Report No. DOE/ER/40614-8

\title{
PROGRESS REPORT FOR GRANT
}

DE-FG06-91ER40614

Particle Physicz - Experimental

August 21, 1991

Jere J. Lord, Prof. of Physics

P. E. Boynton, Prof. of Physics

T. H. Burnett, Prof. of Physics

R. J. Wilkes, Research Prof.

Physics Department FM-15

University of Washington

Seattle, WA 98195

Telephone (206) 543-4230/2770

\section{DISCLAIMER}

This report was prepared as an account of work sponsored by an agency of the United States Government. Neither the United States Government nor any agency thereof, nor any of their bility for the accuracy warranty, express c- implied, or assumes any legal liability or resnonsiprocess disclosed, process disclosed, or represents that its use would not infringe privately owned rights. Refermanufacturer, or otherwise does not product, process, or service by trade name, trademark, mendation, or favoring by the United necessarily constitute or imply its endorsement, recomand opinions of a and opinions of authors expressed herein do not necessarily state or reflect those of the
United States Government or any agency thereof.

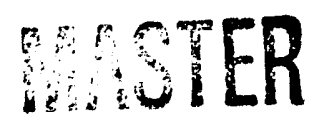




\section{ABSTRACT}

We are continuing a research program in particle astrophysics and high energy experimental particle physics. We have joined the DUMAND Collaboration, which is constructing a deep undersea astrophysical neutrino detector near Hawaii. Studies of high energy hadronic interactions using emulsion chamber techniques were also continued, using balloon flight exposures to ultra-high energy cosmic ray nuclei (JACEE) and accelerator beams (EMU01 Collaboration).

As members of the DUMAND Collaboration, we have responsibility for development and construction of critical components for the deep undersea neutrino detector facility. We have designed and developed the acoustical positioning system required to permit reconstruction of muon tracks with suificient precision to meet the astrophysical goals of the experiment. In addition, we are makix $\mathrm{g}$ signiuicant contributions to the design of the database and triggering system to be used. Deployment of the first components of the detector system is scheduled for 1992. An increasing fraction of our research effort is being devoted to DUMAND.

Work has been continuing in other aspects of the study of multiparticle production processes in nuclei. We are participants in a joint US/Japan program (Project JACEE) to study nuclear interactions at energies two orders of magnitude greater $t\} \cdot n$ those of existing accelerators, using balloon-borne emulsion chambers. We were invited by NASA to pioneer new long-duration balloon flight techniques with our JACEE apparatus. The tests were particularly successful and we obtained two 5 day flights from Australia to South America as well as a record-breaking heavy load flight of 8.5 days from Antarctica. NASA plans to further develop this ballo on flight capability and we have been asked to consider some 10 additional flights over the next 5 years.

On one of the flights we found two nuclear interactions of multiplicity over 1.000 - one with a multiplicity of over 2,000 and pseudorapidity density $\sim 800$ in the central resior. At the statistical level of the JACEE experiment, the froquency of occurence of such events is orders of magnitude too large. We want to follow up this indication of possible new physics at extreme energies. In addition to direct observation of interactions in our detectors, datia collected by JACEE on primary cosmic ray fluxes will provide information on high energy processes in distant galactic accelerators.

We have continued our ongoing program to study hadronic interactions in emulsions exposed to high energy accelerator beams. We are members of a large international collaboration (EMU-01) which has exposed emulsion chamber detectors to beams of ${ }^{32} \mathrm{~S}$ and ${ }^{16} \mathrm{O}$ with energy 60 and $200 \mathrm{GeV} / \mathrm{n}$ at CERN and $15 \mathrm{GeV} / \mathrm{n}$ at Brookhaven National Laboratory. These activities both supplement and complement the balloon-flight program, providing a high-statistics database at lower energies, using the same detector system. 


\section{TABLE OF CONTENTS}

Abstract . . . . . . . . . . . . . . . . . . . . . . . . . . . i

I. DUMAND: Deep Underwater Muon and Neutrino Detector . . . . . . . . . . . 1

A. Physics Motivation and Background

B. Progress to Date

References for Section I

Table I-1

Figures for Section I

II. JACEE: Ultra High Energy Nuclear Interactions . . . . . . . . . . . . . . . . 19

A. Introduction

B. Physics Motivation and Background

C. Experimental Technique

D. Progress to Date

References for Section II

Table II-1

Figures for Section II

III. Accelerator Interactions Studies: EMU-01/E815/E863/E665: . . . . . . . . . . 33

A. EMU01: Physics Motivation and Recent Results

B. Experimental Technique

C. Present Status

D. E665: Present Status

References for Section III

Table III-1

Figures for Section III

APPENDIX I: Publications During the Past Year . . . . . . . . . . . . . . . . . 48

APPENDIX II: Membership Lists for Collaborations . . . . . . . . . . . . . . . 49 


\section{PROJECT DUMAND}

\section{A. PHYSICS MOTIVATION AND BACKGROUND}

In a recent article ${ }^{1}$, Sadoulet and Cronin note that one of the central issues for contemporary particle astrophysics is the nature of the powerful acceleration mechanisms in the vicinity of neutron stars. High energy neutrinos, produced by the same hadronic processes that yield ultra-high energy gamma rays, may provide insight into these mechanisms, and "the DUMAND experiment off the coast of Hawaii represents a first attempt at exploring this virgin territority." Whereas photons may be produced by electromagnetic interactions, neutrinos can only be the products of hadronic interactions, and their presence (or absence) can be used to probe the acceleration process ${ }^{2}$.

The DUMAND Collaboration (Appendix I) has been authorized by DOE to construct a deep underwater laboratory for the study of:

a) high energy neutrino astrophysics, principally the detection of galactic and extragalactic point sources of $\mathrm{TeV}$ neutrinos;

b) particle physics, via indirect observations of UHE hadronic interactions in astrophysical objects as well as more direct observations of terrestrial interactions;

c) cosmic ray physics, relating to muon, primary energy spectrum and composition studies; as well as

d) geophysics and ocean science issues which are confronted incidentally.

A detailed description of the project, a $20,000 \mathrm{~m}^{2}$ effective area deep ocean neutrino detector, is found in the DUMAND II Proposal ${ }^{3}$. The international DUMAND collaboration, from the USA, Japan, and Europe, will construct and deploy this first long term deep ocean array over a period of 2-3 years beginning in 1992.

DUMAND-I consisted of the design, construction and operation of a ship-suspended Short Prototyp: String (SPS) of instruments in November, 1987. Underwater muon fluxes were oboarved to depths of 4000 meters, at a location near the planned DUMAND-II array site ${ }^{4}$. A similar prototype string experiment using Phillips photomultiplier tubes was performed in February, 1991, near the Canary Islands, by European members of the collaboration ${ }^{5}$. All of the necessary technology for the construction of a full array has now been developed and demonstrated in the ocean.

DUMAND-II consists of an octagonal array of 216 high sensitivity photomultiplier tubes, capable of detecting position and direction of single relativistic charged particles with a uniquely large sensitive volume and an angular resolution of 0.5 degrees. The array will be shielded by about $5 \mathrm{~km}$ of water to minimize the incidence of common cosmic ray particles in the detector. The project will be sited in an ocean valley approximately $25 \mathrm{~km}$ west of Keahole Point on the Island of Hawaii, at a depth of $4.8 \mathrm{~km}$. The site has been extensively explored over the last decade, and has been found to be appropriate to our needs.

The overall concept of DUMAND-II is illustrated in Figure I-1. A schematic view of the planned array is pictured in Figures I-2 and 3. Its main properties are summarized in Table I-1. Basically, the proposed design consists of 9 vertical strings, each with 24 photomultiplier detectors spaced $10 \mathrm{~m}$ apart along the string vertically for $230 \mathrm{~m}$, and with the strings spaced $40 \mathrm{~m}$ apart horizontally in an octagonal configuration. This gives a total of 216 detectors, a neutrino induced muon detection solid angle area of $\sim 565,000 \mathrm{~m}^{2} \mathrm{sr}$, and contained mass of 1.8 megatons.

The individual strings are connected to an underwater Junction Box, which is in turn connected to the shore station (containing data processing and power supply equipment) via an electro-optical cable. In addition to optical detector modules (OMs), each string includes an environmental module to provide data 
needed for accurate location of detector elements. The first-stage array of three strings will be deployed in 1992.

The array planned has been optimized for the detection of high-energy inuons from neutrino interactions. Calculations indicate that this gives us the best opportunity for detecting; extraterrestrial neutrino sources. The detector spacings, $40 \mathrm{~m}$ horizontally and $10 \mathrm{~m}$ vertically, ensure that rauons with energy $\geq 25 \mathrm{GeV}$ which pass through from outside, will be detected with high efficiency and reconstructed in direction with better than $1 \mathrm{deg}$ accuracy. The original neutrino direction will be within this error for neutrino energies above about $1 \mathrm{TeV}$.

The array will count downward-going cosmic ray muons at the rate of about one muon per 20 seconds, or $4.6 \times 10^{6}$ events per year, of which about two percent will be simultaneous multiple muons. Muons from atmospheric neutrinos (the decay products of secondary cosmic rays produced in the atmosphere) will dominate the down going muons beyond a zenith angle of about $80 \mathrm{deg}$, leaving a solid angle of $2.35 \pi$ sr for neutrino observations. The rate of cosmic ray neutrino induced muon events will be about $10^{-4} / \mathrm{sec}$ or $3500 /$ year. This gives a background of about one atmospheric neutrino per $(2.8 \mathrm{deg})^{2} /$ year. The full DUMAND array will be about two orders of magnitude more sensitive than previous detectors located underground.

We expect detectable signals from a number of point sources both inside and outside the galaxy, and from the diffuse background of neutrinos produced by cosmic rays passing through the denser portions of our galaxy. Fig. I-4 shows the expected event rates from a number of source candidates, making reasonable assumptions based on measured or estimated photon rates and the ratio of neutrino to photon fluxes.

All of the figures given above were based upon conventional estimates of the high energy neutrino Alux. However, Stecker and collaborators recently described a new model ${ }^{6}$ for ultrahigh energy neutrino production in Active Galactic Nuclei (AGN). Taking into account the summed flux from all AGNs, their calculations suggest that for muon energies above about $50 \mathrm{GeV}$, AGNs may be the dominant source of neutrinos. Estimated muon detection rates in DUMAND for atmospheric and AGN neutrinos are shown in figure I-5a as a function of detected muon energy; in figure I-5b we show the expected zenith angle distribution ${ }^{7}$. Note that atmospheric and AGN neutrinos have similar angular distributions for different reasons: the atmospheric $\nu$ rate peaks near the horizon due to the pion decay path distribution, while the AGN rate is depressed at the nadir because the very high mean energy causes significant attenuation in the earth. If the fluxes predicted in ref. $[6]$ are observed, we would expect about 3500 neutrinos events per year from AGNs, nearly half with energy over $10 \mathrm{TeV}$ at the detector.

In addition to opening new territory in astrophysics and astronomy, DUMAND will complement accelerator based high energy physics research. Currently existing or planned accelerator facilities offer no neutrino beam above about $600 \mathrm{GeV}$, while DUMAND explores energies above $1 \mathrm{TeV}$. The rising neutrino cross section and the enormous effective volume of DUMAND offers the best available oppurtunity to continue the very fruitful study of neutrino interactions energies above $1 \mathrm{TeV}$ where significant $W$-boson propogator and QCD effects are expected. The AGN model mentioned above also predicts substantial $\nu_{e}$ fluxes which may permit the observation ${ }^{7}$ of resonant $W^{-}$production at $6.4 \mathrm{PeV}$.

Measurement of the neutrino induced muon angular distribution (with particular sensitivity to the region near the horizon), will give a neutrino mass sensitivity in the range $0.01 \leq \delta \mathrm{m}^{2} \leq 100 \mathrm{eV}^{2}$ for mixing angles $\sin ^{2}(2 \theta) \geq 0.1$. Five years of data from DUMAND will result in a factor of five improvement in statistical precision over the sum of all the present and planned underground experiments.

We have submitted a proposal to Fermilab (P824) which requests that construction of the new $150 \mathrm{GeV}$ 
proton injector include facilities for directing a neutrino beam toward the DUMAND site to permit long baseline neutrino oscillations studies ${ }^{8}$. The path length is $6482 \mathrm{~km}$, which allows us to probe a much lower range of $\delta m^{2}$ than proposed experiments using existing underground facilities in the continental US. For the proposed Fermilab beam, $\langle E\rangle=20 \mathrm{GeV}$ and DUMAND-II should be able to reach down to $\delta m^{2}<10^{-3}$ $\mathrm{eV}$. When the expected spectral shape of the $\nu$ beam is taken into account, the effective target mass for fully fitted $\nu_{\mu} \rightarrow \mu X$ events is $3.1 \times 10^{6} \mathrm{~kg}$. Furthermore, matter oscillation effects (due to the fact that only $\nu_{e}$ may forward scatter via the charged weak current) will greatly enhance the sensitivity to small mixing angles for $\nu_{\mu} \rightarrow \nu_{e}$ oscillations ${ }^{9}$. Taking into account matter oscillations effects, it should be possible to reach down to mixing levels of $\sin ^{2} \theta \sim 10^{-2}$. Fig. I-6 shows the parameter ranges available to DUMAND-II/P824.

No more important issue for cosmology and particle astrophysics exists than the nature of the missing "dark matter", and DUMAND should be able to make unique contributions to that search. It must be emphasized that DUMAND will be unmatched by any other detector in the world for sheer area and volume. The catalysis of baryon decays by magnetic monopoles presents an outstanding current example. This process would give an easily recognizable signature in DUMAND. The recent history of high energy theory indicates that predictions of this sort come along every few years, and some of the objects or processes predicted may be observable in DUMAND. In searching for rare phenomena, DUMAND will be capable of setting limits several orders of magnitude better than any other detector.

DUMAND can provide information on the origins and acceleration mechanisms of the primary cosmic rays. It provides a new data channel that complements existing ground-level cosmic ray detectors at the upper end of the energy spectrum, such as CASA and the "Fly's Eye." The measurement of the muon energy distribution via the angular distribution of down-going muons, and observation of the spectrum of electromagnetic bursts caused by down-going muons will enable us to study the spectrum up through the UHE range.

\section{B. PROGRESS TO DATE}

Our group joined the DUMAND Collaboration in May, 1990 and immediately began work on an aspect of the project that required additional effort urgently: final design, development and construction of the Environmental Modules, both for the Junction Box and for the individual strings.

During array operation, small ocean bottom currents will cause slow (time scale $\sim$ hours) variation of string position. The anticipated maximal horizontal excursion is $5 \mathrm{~m}$ at the top of the string, less than ore degree off the vertical, with all strings tilted approximately in parallel; the array will behave like an artificial kelp bed. We plan to monitor string position dynamically by acoustical triangulation, which imposes stringent requirements on our knowledge of parameters affecting the local sound velocity, such as temperature, salinity and pressure, as well as string tilt and orientation.

An Environmental Module (EM) will therefore be deployed on each string, as well as on the Junction Box (JB). Each EM will have hydrophone and tiltmeter instrumentation. Water temperature, pressure, and salinity sensors are provided in standard commercial oceanographic instrument packages called CTDs. Two of these units will be used in the initial three-string deployment, one on the JB, and one on a string; a third will be deployed on one of the 6 strings installed in the second phase of operation. Finally, the JB environmental module will be equipped with video and illumination systems to aid in initial placement and in making interconnections, as described below. A block diagram of the JB environmental module is shown in Figure I-7, and the physical layout of the JB string is shown in Figure I-8.

The OM spacings have been designed to ensure that muons will be detected with high efficiency and 
reconstructed in direction with a median accuracy of about $1^{\circ}$. The scattering angle between the incident neutrino and the resulting muon will be within this error for neutrino energies above about $1 \mathrm{TeV}$. Thus it is important that the positions of the optical modules be known with sufficient precision to ensure $1^{\circ}$ accuracy in reconstruction of tracks. This requires determination of $O M$ positions to better than $10 \mathrm{~cm}$, placing a stiff specification on the acoustical positioning system which will be used to achieve this goal. With conventional deep-ocean positioning techniques it would be difficult to determine positions reliably to better than $\sim 0.5$ m.

In addition to fixing the relative positions of the individual OMs, it will be necessary to survey the site orientation to better than $1^{\circ}$ relative to geographical north in order to project neutrino directions onto the celestial sphere with appropriate precision to meet astrophysics requirements.

At the time of Junction Box placement we will deploy 4 battery powered acoustic transponders around the array, about $1 \mathrm{~km}$ from the array center, to define the site location for further operations, and to provide a baseline for orientation of site coordinates relative to celestial coordinates. The transponders will be located to $<10 \mathrm{~m}$ accuracy from a surface ship using GPS (Global Positioning System satellite) navigation, and will serve as site fiducials for inter-relating other components. These units are designed to reply when triggered by a coded acoustic pulse. Initial plans were to use conventional transponders in conjunction with a pinger and sets of hydrophones on each string for the array positioning task. Sound speed parameters would be determined by the CTDs and a current meter near tive array, and conventional transponder ranging techniques would be used to determine hydrophone coordinates relative to the surveyed transponder array.

However, typical maximum transponder battery lifetime would be insufficient for the needs of the experiment, requiring replacement and resurvey. The anticipated running period of 10 years exceeds even the standby lifetimes of typical transponder battery systems; since array motion cannot be precisely predicted, positioning pings may be required as often as once per minute, or 500,000/yr. Moreover, a transponder suitable for site survey purposes at $5000 \mathrm{~m}$ depth would be limited to frequencies on the order of $10 \mathrm{KHz}$, while positioning to $\approx$ few $\mathrm{cm}$ would require $>50 \mathrm{kHz}$ using conventional techniques. The presence of the shore cable supplying power and high-rate data communications makes a different approach practical. Instead of autonomous battery powered transponders, we can use hardwired units (called responders), with signals commanded from shore rather than acoustically triggered. Sound speed parameters become relatively unimportant since we can calibrate local sound speed by sing-around procedures using the surveyed responder spacings.

The fact that signals can be digitized at high sampling rates and sent to shore for analysis means that we are not limited to the simple threshold detection methods used in transponders, but can employ more sophisticated digital convolution techniques to obtain enhanced positioning precision ${ }^{10}$. The possibilities for further development of these positioning techniques are unique to DUMAND and technically exciting. In fact, we have shown that the chirping (frequency modulated pinging) technique we plan to use provides positioning accuracy on the order of $1 \mathrm{~cm}$, and extremely high signal extraction capability in the presence of noise. This permits relatively low power projectors (output transducers, the aquatic version of loudspeakers) to be used. We plan to deploy 4 responders (hardwired, computer-commanded transponder units) at a range of about $300 \mathrm{~m}$ from the DUMAND array. The deployed units will contain only transducers, with all electronics located at the Junction Box (JB). Also suspended from the JB will be a projector and an array of three hydrophones with fixed, well-defined spacings, to supplement the outlying set of responders.

Most commercial positioning systems use relatively straightforward leading edge detection on pings (i.e., constant frequency pulses). This imposes a tradeoff between prccision and usable range. To get $5 \mathrm{~cm}$ 
precision on positions, one needs to determine pulse arrival times to better than 0.03 msec. If the leadingedge discrimination system requires at least 2 cycles of signal, frequencies $>60 \mathrm{kHz}$ must be used. At these frequencies, usable range would limited to about $1 \mathrm{~km}$, insufficient to reach the ocean surface fom $5000 \mathrm{~m}$. In contrast, convolution techniques, especially when applied to frequency modulated signals as described below, permit extremely precise pulse timing, at significant cost in signal processing. Since all DUMAND hydrophone signals will be digitized and sent to shore anyway, the available signal processing capability is, in practical terms, unlimited. Thus we can use feature extraction techniques which would be very difficult to implement if all signal processing had to be done on the seabed.

Figure $9 \mathrm{a}$ shows a simulated $15 \mathrm{kHz}$ ping, and Fig. 9b shows the same pulse with gaussian noise superimposed. While signal extraction with simple threshold logic would be difficult, Figure 9c shows how the signal can be clearly seen by convolving the raw data with a replica of the pure signal. However, the timing resolution, measured by the sharpness of the peak in the correlogram, is still equivalent to several periods, corresponding to $>20 \mathrm{~cm}$ spatial resolution. Fig. 9d shows a frequency-modulated chirp (10-30 $\mathrm{kHz}_{\text {j }}$ and Fig. 9e shows the chirp with superimposed noise as in Fig. 9b. The results of replica convolution are shown in Fig. 9f. Notice the extremely sharp time resolution of the correlogram. Fig. 9f demonstrates how the FM chirp correlation technique can extract clean timing data from data with a very poor $\mathrm{S} / \mathrm{N}$ ratio.

A prototype chirping system has been constructed and tested. Fig. 10 shows data logged from a test in which projector and hydrophone were separated by $100 \mathrm{ft}$ in fresh water. Results indicate that the spacing could be determined to $\sim \mathrm{cm}$ precision over this range ${ }^{11}$. Further testing, over longer ranges and in seawater, is being carried out now.

The plan for DUMAND positioning involves three sets of equipment at the site: 4 responders (computercontrolled projector/hydrophone sets) on short tethers, deployed radially approximately $300 \mathrm{~m}$ from the OM strings; sets of hydrophones (5 per OM string); and transducers on the JBEM string (a projector and a set of 3 rigidly spaced hydrophones). The responders and the JBEM will be surveyed in absolute geographic coordinates by a surface vessel with GPS navigation, as part of the deployment process. These units serve as benchmarks both to determine the internal relative coordinate system of the DUMAND array, and to relate that cooidinate system to celestial coordinates via the temporary transponder array laid at $1 \mathrm{~km}$ during deployment. The responders will chirp to the string hydrophones, providing range measurements for OM positioning. The responders will also be ranged relative to one another and to the JBEM string, providing internal local sound speed determination independent, of CTD data.

The hardwired responder design includes the capability for downloading new chirp profiles from shore, so it will be possible to undertake studies of positioning accuracy and efficiency versus modulation scheme, frequency range, analysis mode, and other variables. The responder positions will be known accurately following the initial survey, and inter-responder positioning can be used as a testbed.

Each string will be equipped with a set of five hydrophones, with 4 units spaced equally over the upper $240 \mathrm{~m}$ of the string (where the $\mathrm{OMs}$ are located) and the fifth near the bottom. The hydrophone positions, determined by simultaneous ranging from the surveyed fiducial responders, will be fitted to an appropriate catenary function to interpolate OM coordinates on each string.

The hydrophones will be interfaced with an environmental module placed at the center of each string, in the String Controller electronics canister. Only two full EM units are required, providing precise temperature, pressure and salinity for determination of the local speed of sound, as well as processing hydrophone data. Reduced instrumentation on the remaining strings will monitor the azimuthal orientation and tilt to provide redundant information on string motion. We selected a 68000 -based single board microcomputer, 
the GESPAC MPL-4080, to serve as the controller for the JB EM package. This selection influenced the collaboration as a whole, and the device we suggested will also be used by other subsystem developers within the collaboration.

We will are also responsible for constructing a television monitoring system for the JB string, required to aid in deployment operations. The video system will consist of one fixed camera, aimed at the connector array on top of the JB, and one camera mounted on a shore-controlled tilt/pan/zoom unit. Thallium-iodide lamps will provide illumination optimized for the camera sensitivity and water transparency spectra.

We will digitize the hydrophone data at a $100 \mathrm{kHz}$ rate, and hydrophones will provide response between 5 and $50 \mathrm{kHz}$. Thus the acoustical positioning data stream will also yield useful information for exploring the deep ocean high frequency (up to $50 \mathrm{kHz}$ ) acoustic background, and allow us to explore the possibility of using sound pulses in a future very large $\left(100 \mathrm{~km}^{3}\right.$ range) and cost-effective acoustic array ${ }^{12}$ for observing interactions in the range above $10^{16} \mathrm{eV}$. DUMAND will provide additional oceanographic data of interest, such as long-term measurement of currents and other physical parameters, and information on bioluminescence (seasonal fluctuations, diurnal variation, spatial correlations, etc.) reflected in OM backgrounds. Correlations between environmental observations in different modes (acoustical, physical and optical) may provide exciting new insight into the abyssal environment. If the AGN flux model is valid, event rates might be high enough to permit a $5 \%$ determination of the earth's core density in one year, from the angular distribution.

We have had substantial assistance from our colleagues in the University of Washington Applied Physics Laboratory (APL) and the Department of Oceanography, who have long experience with deep underwater technology. APL has provided a considerable amount of informal consulting and equipment loan during the development effort. In addition to our University colleagues, DUMAND has excited considerable interest in the large local community of ocean engineers and scientists, and we have established contact with several private firms in the Seattle area with extensive experience in producing commercial systems similar to the EM modules required for DUMAND. These resources are being utilized as required during the EM design, construction and testing process.

Additional, less time-critical areas in which we are contributing include design of a suitable experimental database system, development of triggering systems, definition of astrophysical source candidates and statistical analysis procedures, and participation in the major software effort required Sor data acquisition, reduction and analysis. Our group members have substantial and recognized expertise in these areas. In July, 1990, we served as host institution for a DUMAND Trigger Subgroup Workshop. Members of our local group made essential contributions to the design of the data transfer system to be used by the trigger processor $^{13}$. The MPP (Massively Parallel Processor) Collabcration at CERN, which is developing arrays of Associative Processor Elements (APEs) for triggering and pattern recognition ${ }^{14}$, has expressed interest in working with us on possible advanced triggering system designs. We are also working on development of a database design for DUMAND in cooperacion with collaborators at Hawaii and Aachen. 


\section{REFERENCES for Section I:}

1. B. Sadoulet and J. W. Cronin, Physics Today, April, 1991.

2. H. W. Sobel, invited paper at Neutrino-90 Conference, Nuclear Physics B19, 444 (1991).

3. DUMAND-II Proposal, U. Hawaii document HDC-2-88 (1988). For recent updates, see also: V. Peterson, et al, U. of Hawaii preprint HDC-7-91, Proceedings of the OCEANS-91 Conference, to be published, and R. J. Wilkes, et al, U. of Washington preprint UWSEA-91-09, Proc. 4th Conf. on Interseciions of Particle and Nuclear Physics, to be published by American Institute of Physics, New York.

4. J. Babson, et al, Physical Review D42, 3613 (1990); S. Matsuno, et al, N.I.M., A276, 359 (1989).

5. P. Bosetti, private communication; S. O. Flyckt, Proc. DUMAND Optical Module Workshop, p. 4, Tohoku University Report, 12/90.

6. F. W. Stecker, C. Done, M. H. Salamon and P. Sommers, "Ultrahigh Energy Neutrinos from Active Galactic Nuclei and their Detectabilityn, Univ. of Utah preprint (to be published in Proc. of Srd Int. Workshop on Neutrino Telescopes, Venice, 1991).

7. J. G. Learned and T. Stanev, “Detection of Neutrinos from Active Galactic Nuclei”, Univ. Hawaii Preprint HDC-1-91, (to be published in Proc. of Srd Int. Workshop on Neutrino Telescopes, Venice, 1991). 8. A. Roberts and M. Webster, et al, Letter of Intent and Fermilab Proposal P824.

9. J. Pantaleone, Phys. Lett. B246, 245 (1990); V. Stenger, U. Hawaii preprint HDC-4-91; V. Stenger, et al, Paper 5.3.6, Proc. XXII International Cosmic Ray Conference (to be published, Trinity College, Dublin, 1991), U. Hawaii preprint HDC-8-91 (1991).

10. R. J. Wilkes, et al, "Acoustical Positioning System for DUMAND-II", Paper 5.3.5, Proc. XXII International Cosmic Ray Conference (to be published, Trinity College, Dublin, 1991); UW preprint UWSEAPUB-91-14 (1991). S. Schock, et al, Proc. 18th Offshore Technology Conf., p. 389 (1986).

11. B. Egaas, et al, U. of Washington internal report VTL-ON-148, 1991; see also ref. 10.

12. J. G. Learned, Phys. Rev. D19, 3293 (1979).

13. Proc. 1990 DUMAND Trigger Workshop, K.K. Young and R.J. Wilkes, eds., Univ. of Washington Report (IWA-90-2, 1990.

14. F. Rohrbach, CERN Report DRDC/90-76, January, 1991. 
Table I-1: Summary of DUMAND-II Array Characteristics

\begin{tabular}{|c|c|}
\hline $\begin{array}{l}\text { Depth of sea floor } \\
\text { Array dimensions } \\
\text { Number of strings in array } \\
\text { Horizontal string spacing } \\
\text { Vertical sensor spacing along string } \\
\text { Number of optical sensors/string } \\
\text { Total number of optical sensors (OMs) } \\
\text { Height of first OM } \\
\text { OM pressure envelope } \\
\text { Optical sensors } \\
\text { Acoustical transducers }\end{array}$ & $\begin{array}{l}4.8 \mathrm{~km} \\
105 \mathrm{~m} \text { diameter, } 230 \mathrm{~m} \text { high } \\
\text { Vertices of octagon }+1 \text { in center } \\
40 \mathrm{~m} \text { side, } 50 \mathrm{~m} \text { to center } \\
10 \mathrm{~m} \\
24 \\
9 \times 24=216 \\
100 \mathrm{~m} \text { above sea floor } \\
17^{n}(43.2 \mathrm{~cm}) \text { O.D., glass } \\
15^{n} \text { Hamamatsu and Phillips PMTs } \\
5 \text { hydrophones per string } \\
3 \text { hydrophones }+1 \text { projector } \\
\quad \text { on Junction Box } \\
4 \text { responders (hydrophone + projector) } \\
\quad 300 \mathrm{~m} \text { from array } \\
12 \text { optical fibers ( } 625 \mathrm{Mbps} \text { each) } \\
5 \mathrm{~kW} \text { electrical power (350VDC at site) }\end{array}$ \\
\hline $\begin{array}{l}\text { Contained volume of array } \\
\text { Target area for through-going } \\
\text { muons } \\
S \Omega \text { for } \nu \text {-induced muons } \\
\text { Effective target volume for } 2 \mathrm{TeV} \mu \text { 's } \\
V_{\text {eff for } 1 \mathrm{TeV} \text { cascades }} \\
\text { Muon energy threshold } \\
\text { Track reconstruction accuracy } \\
\text { Cascade detection threshold } \\
\text { Muon rate, down-going } \\
\text { Atmospheric neutrino detection: } \\
\quad \text { Rate for through-going muons } \\
\quad>25 \mathrm{GeV}: \\
\quad>1 \mathrm{TeV} \text { : } \\
\text { Rate for contained events }>1 \mathrm{TeV} \\
\text { Sensitivity (>1 TeV, in } 1 \mathrm{yr} \text { ): } \\
\text { Point sources } \\
\text { Contained events } \\
\text { Assuming AGN fluxes of Stecker, et } 2 l \text { : } \\
\text { AGN muons }>25 \mathrm{GeV} \\
\text { AGN } \nu_{e} \rightarrow \text { cascade (contained) }\end{array}$ & $\begin{array}{l}1.8 \times 10^{6} \mathrm{~m}^{3} \\
23,000 \mathrm{~m}^{2} \text { horizontal } \\
7,850 \mathrm{~m}^{2} \text { vertical upward } \\
2,500 \mathrm{~m}^{2} \text { vert. downward } \\
565,000 \mathrm{~m}^{2} \mathrm{gr} \\
1.0 \times 10^{8} \mathrm{~m}^{3} \\
7.0 \times 10^{5} \mathrm{~m}^{3} \\
10 \text { to } 50 \mathrm{GeV} \\
1.0^{\circ} \text { median } \\
\sim 10 \mathrm{GeV} \\
3 \text { per minute }\end{array}$ \\
\hline
\end{tabular}


Figures for Section I:

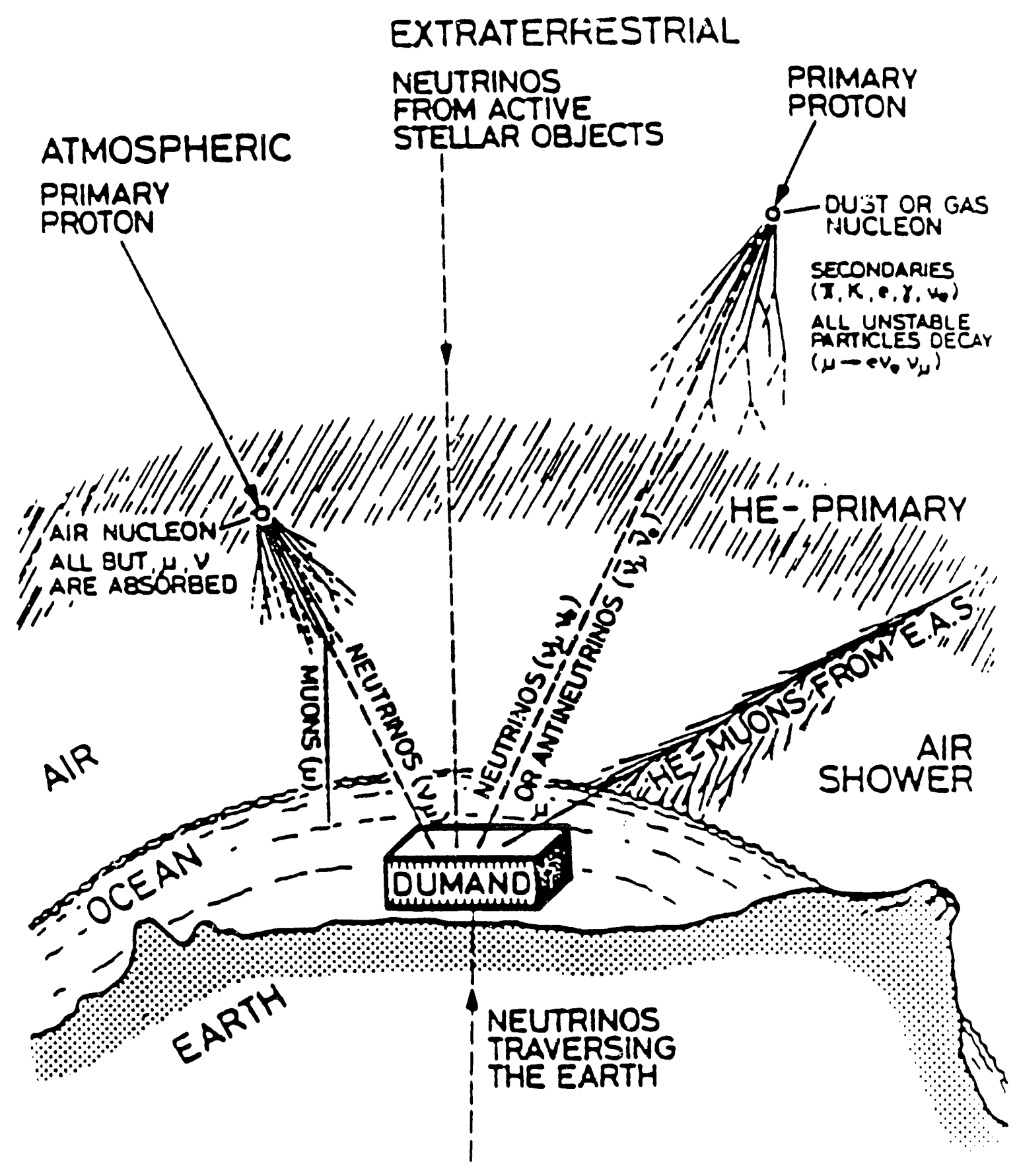

1-1. Concept of the DUMAND-II astrophysical neutrino detector. 


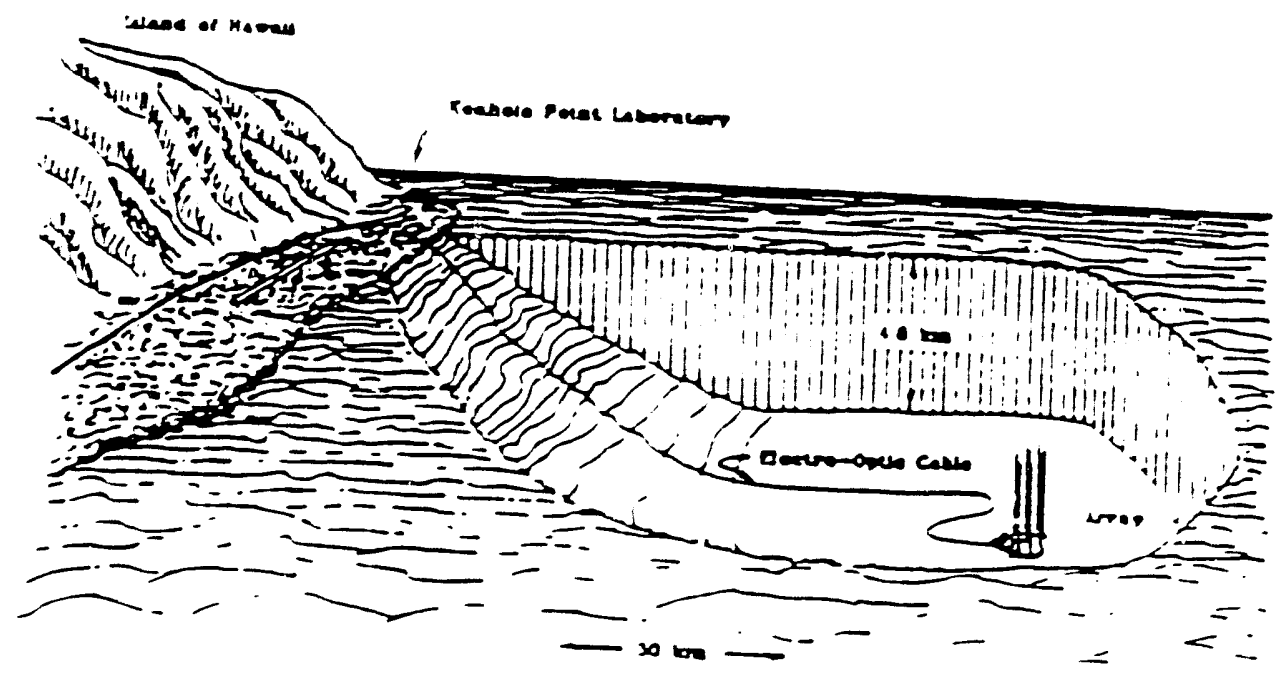

1-2. Schematic view of the DUMAND-Il array, shore cable, and shore station. 


\section{DUMAND-II: Deep Underwater Muon and Neutrino Detector}

Located $32 \mathrm{~km}$ west of Keahole Point, Hawaii

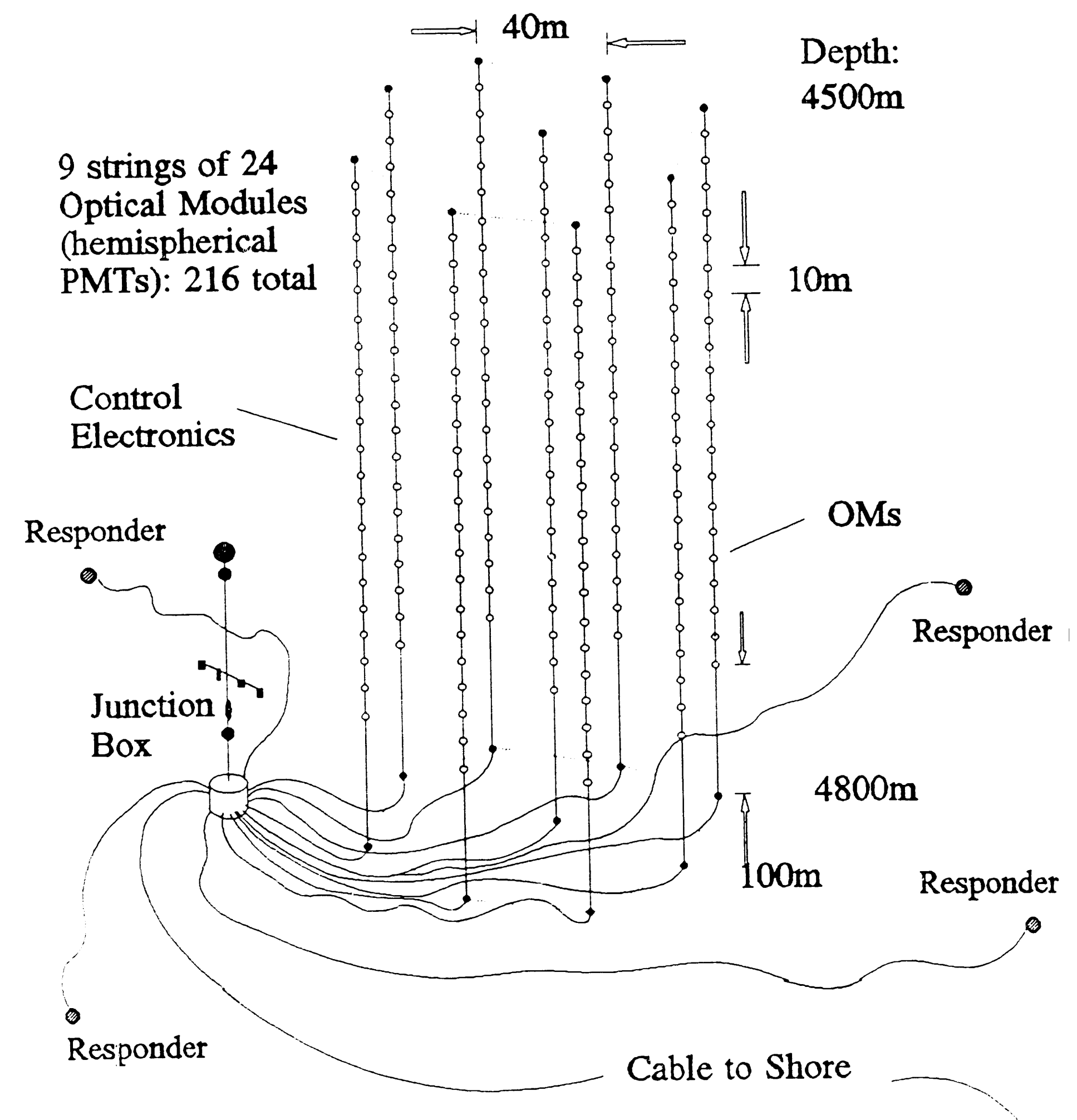

I-3. Schematic view of the 9-string array, showing Junction Box and acoustical positioning syistem compo- 


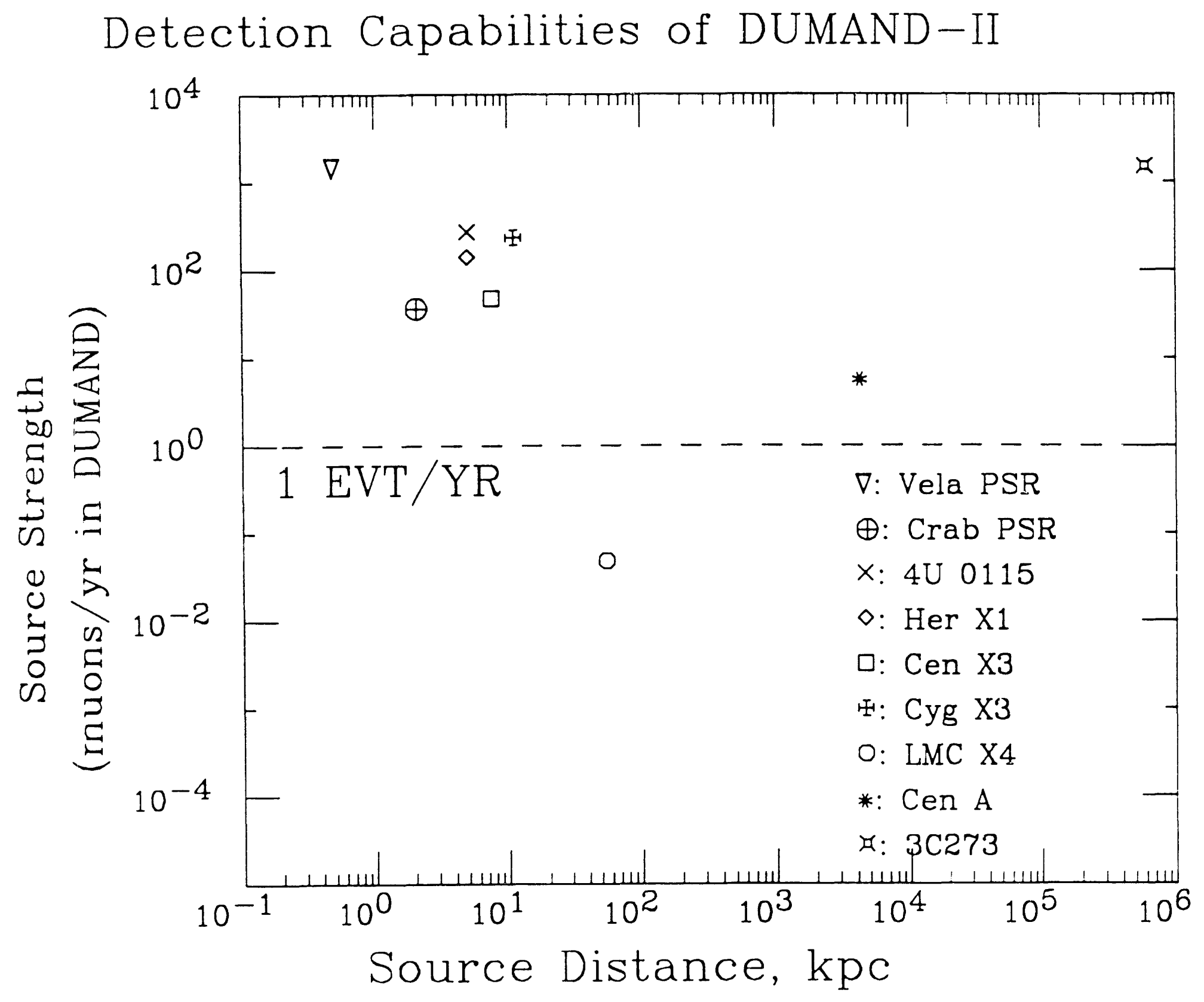

1-4. Estimated event rates (muons per year) for selected neutrino point source candidates. 
DLMAND-II: Integral spectrum of upcoming muons
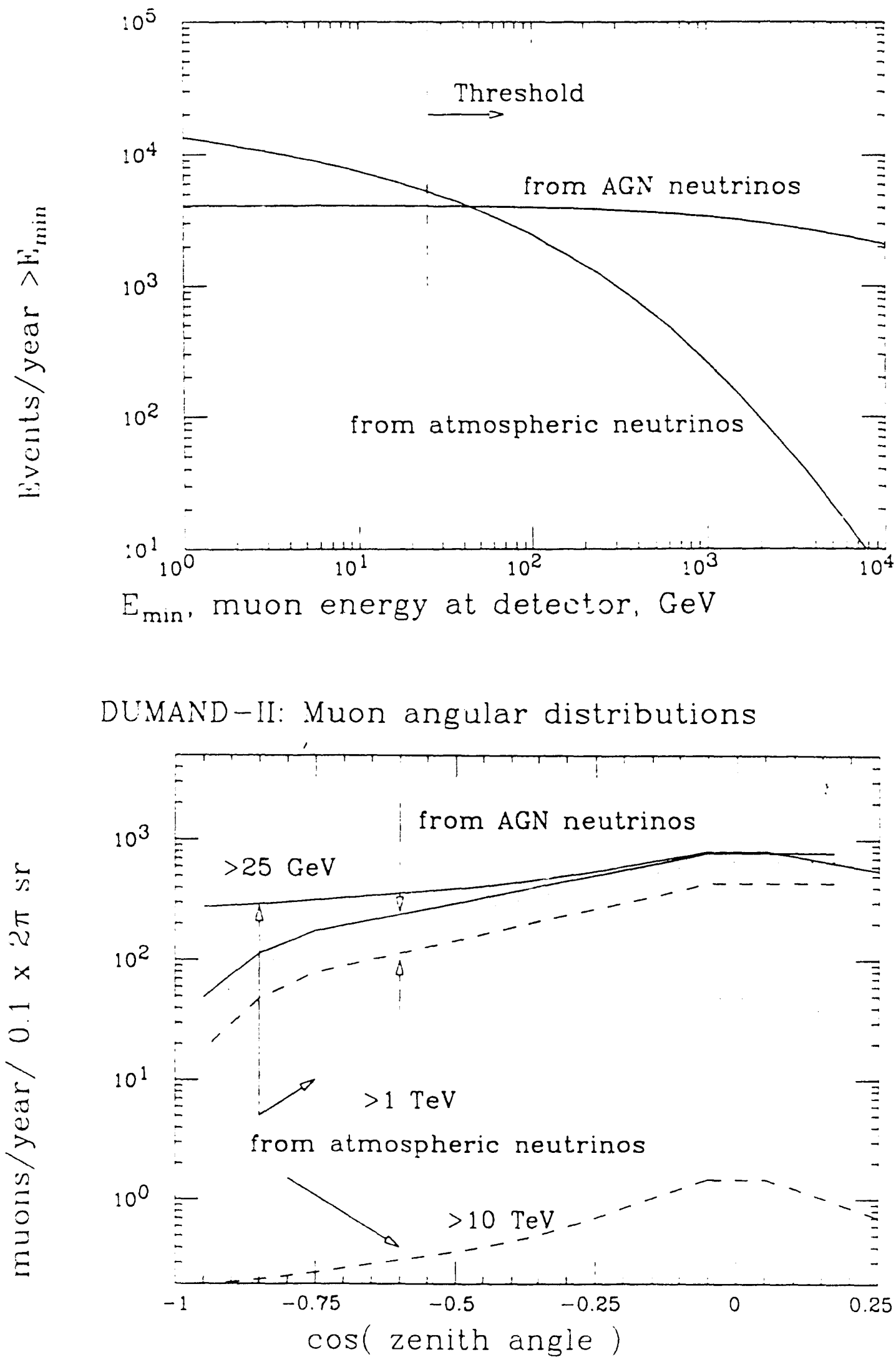

1-5. Calculated muon rates in the DUMAND-II array from atmospheric and ACX neutrino whis as a function of the a) muon energy; b) zenith angle $\theta_{\mu}$, based on the model of Stectier. Donte and Salannon. 


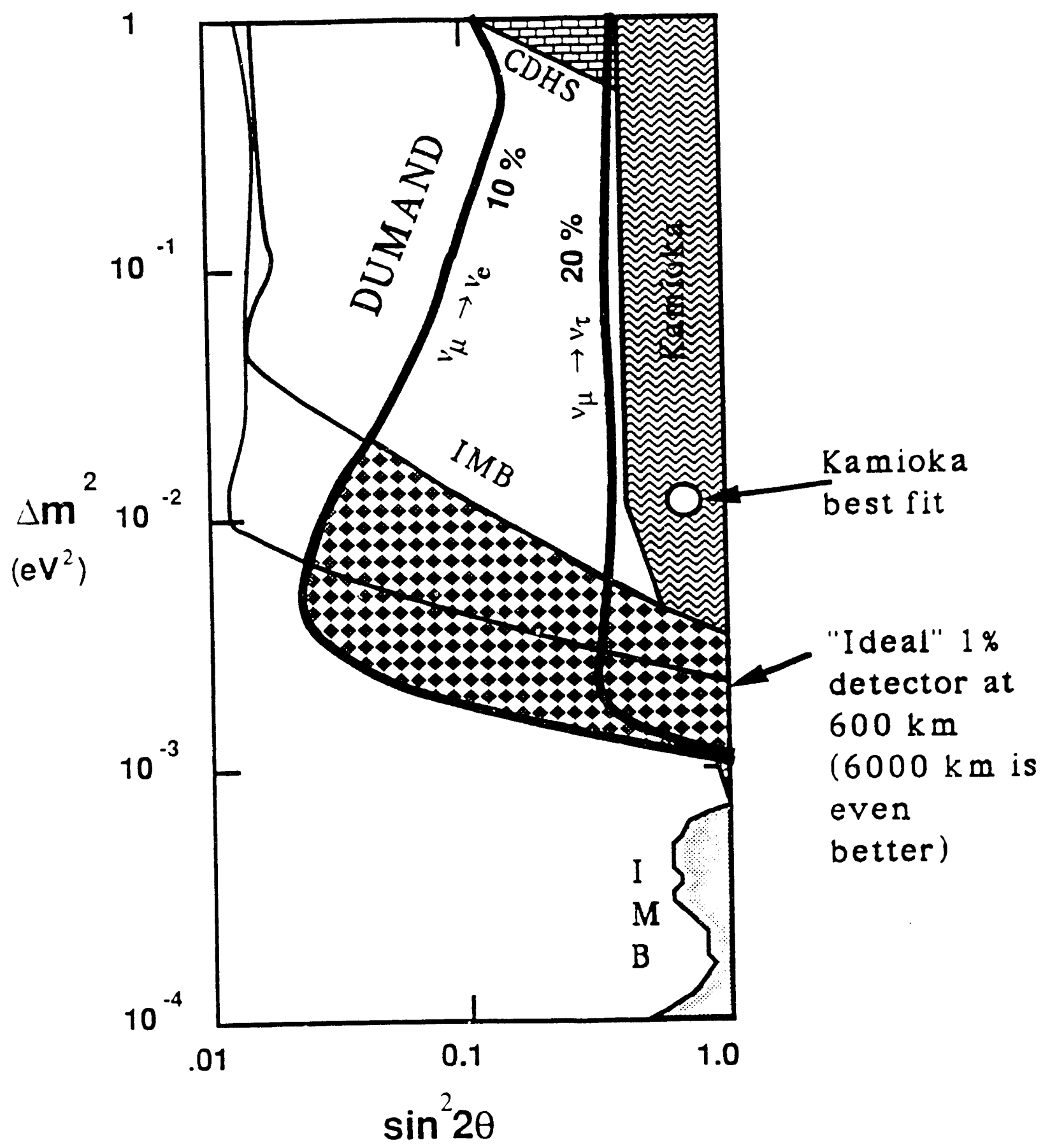

1-f i heckered area shows sensitive region in $\delta m^{2}$ and $\sin ^{2} \theta$ for DCMAND. assuming directed beam from Fermulab. 


\section{JBEM CONTROLLER DIAGRAM}

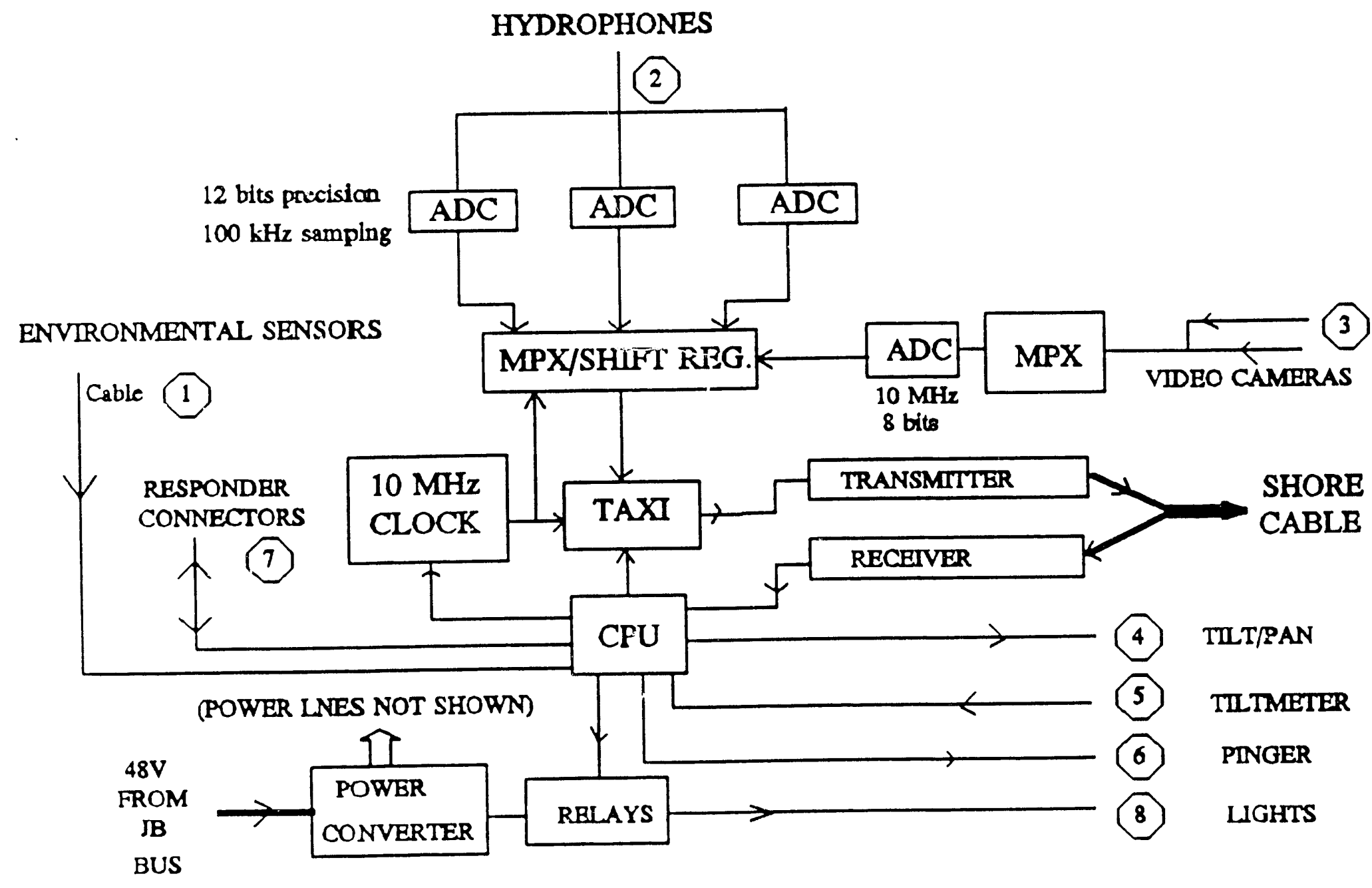




\section{JBEM PHYSICAL LAYOUT}

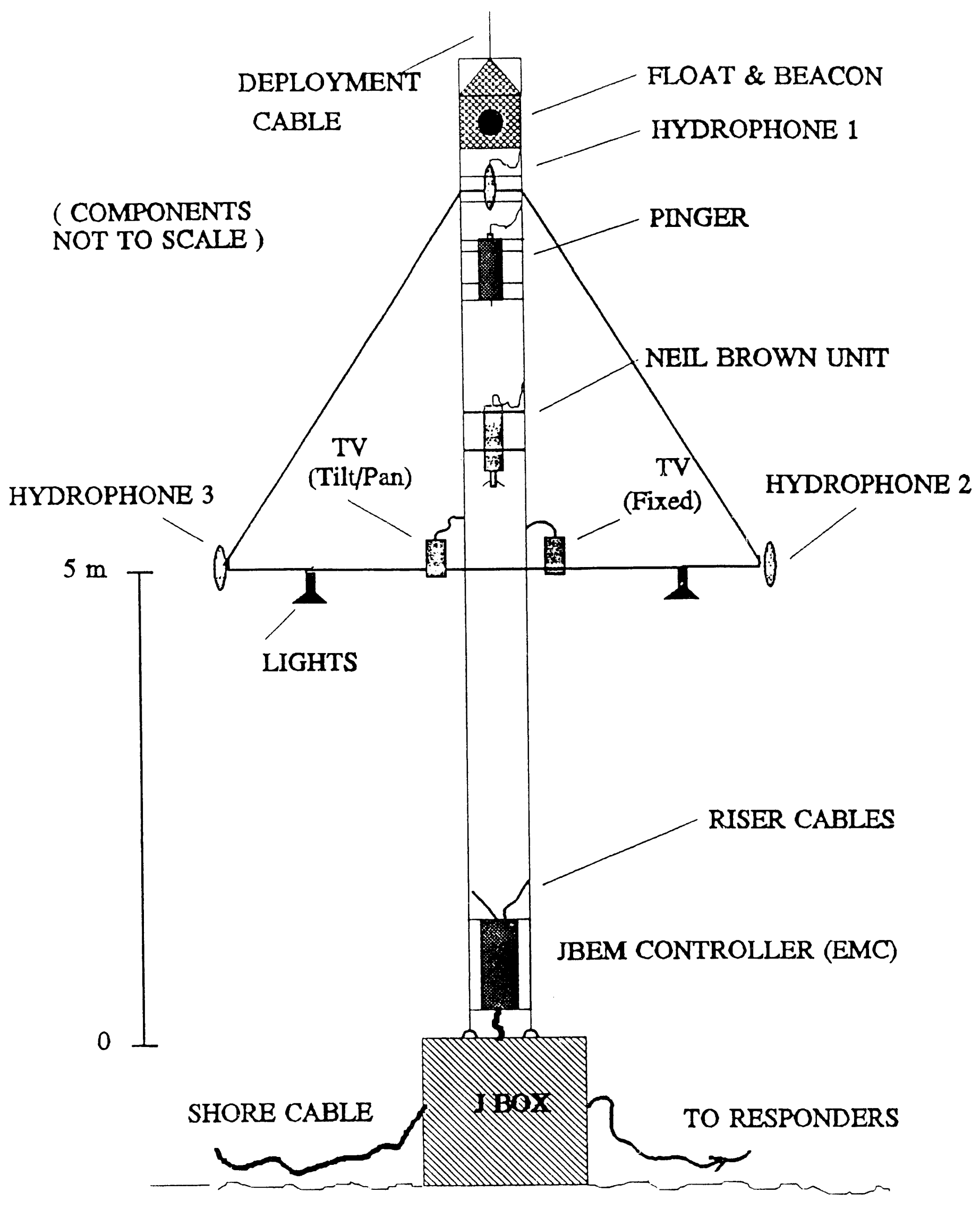

1-8. Physical layout of Junction Box Environmental string. 
(a.) Ping: $15 \mathrm{kHz}, 4 \mathrm{msec}$
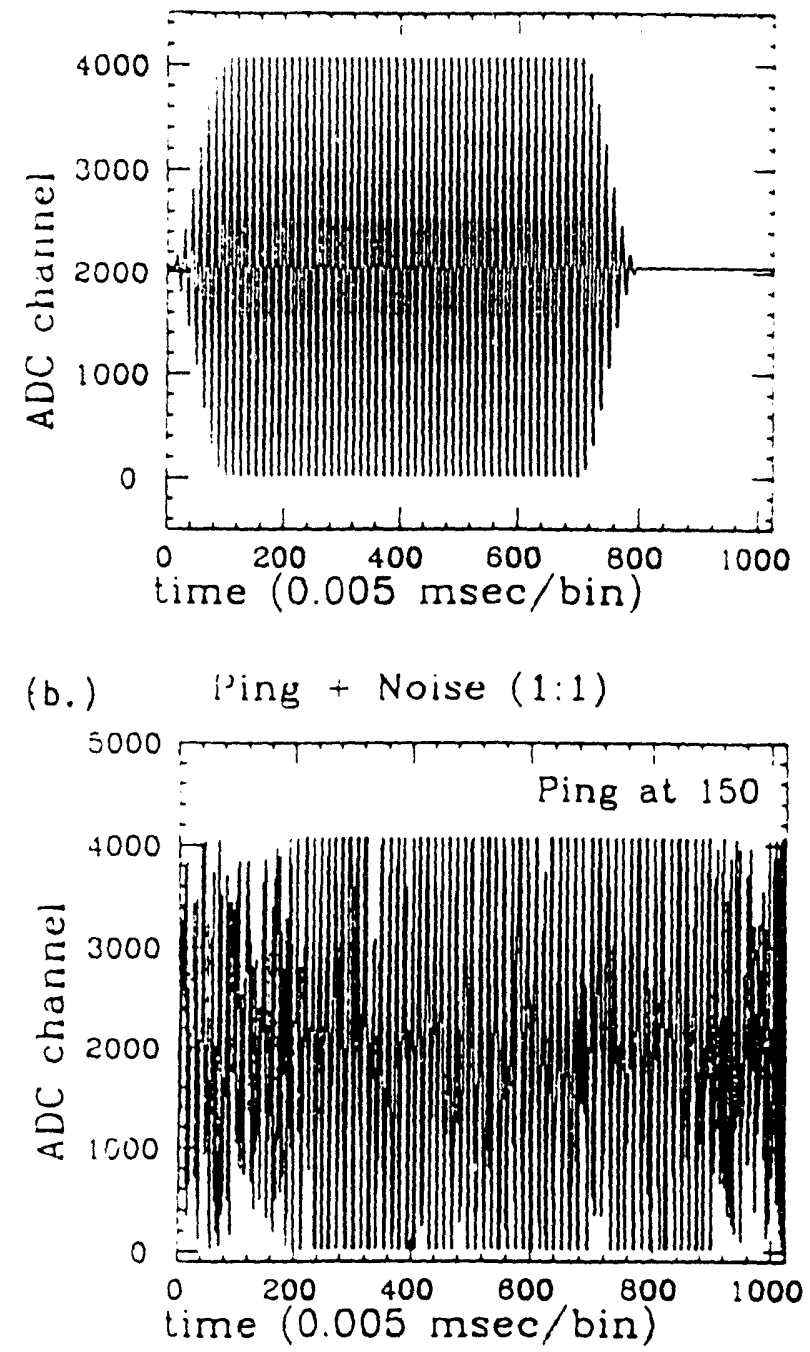

(c.) Correlalion on Noisy fing

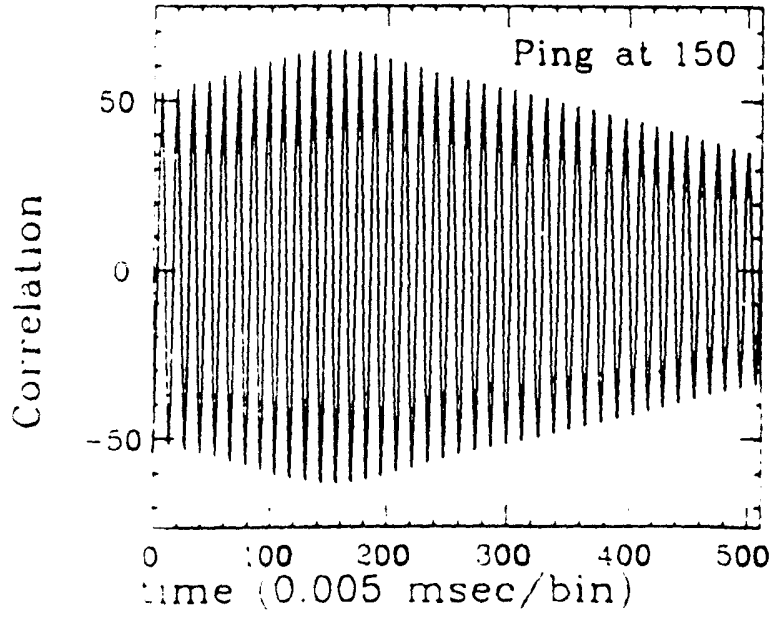

(d.)

Chirp: $: 0-30 \mathrm{kHz}$

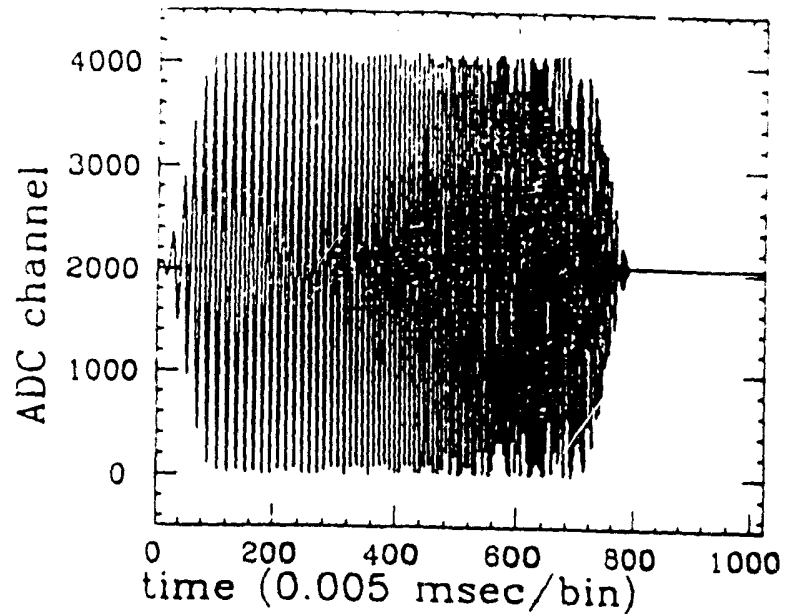

(e.) Chirp + Noise (1:1)

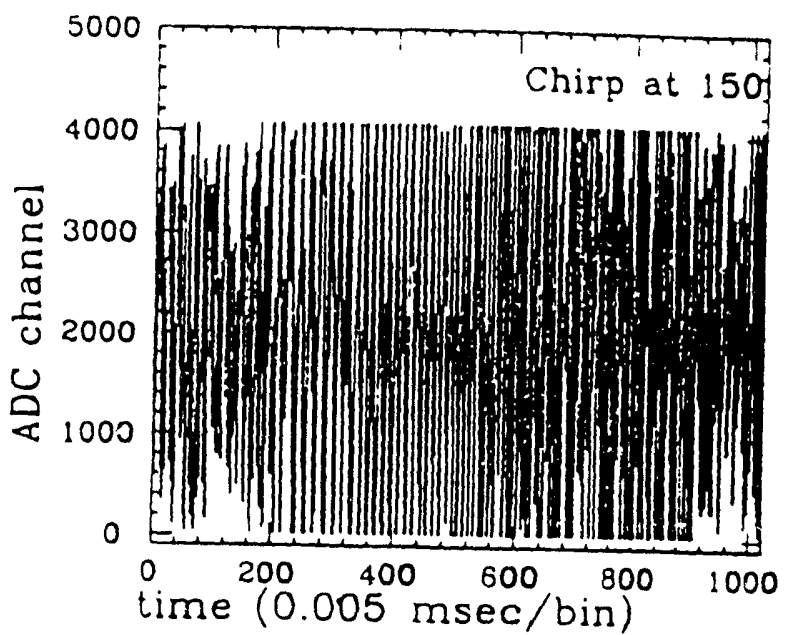

(f.) Correlation on Noisy Chirp

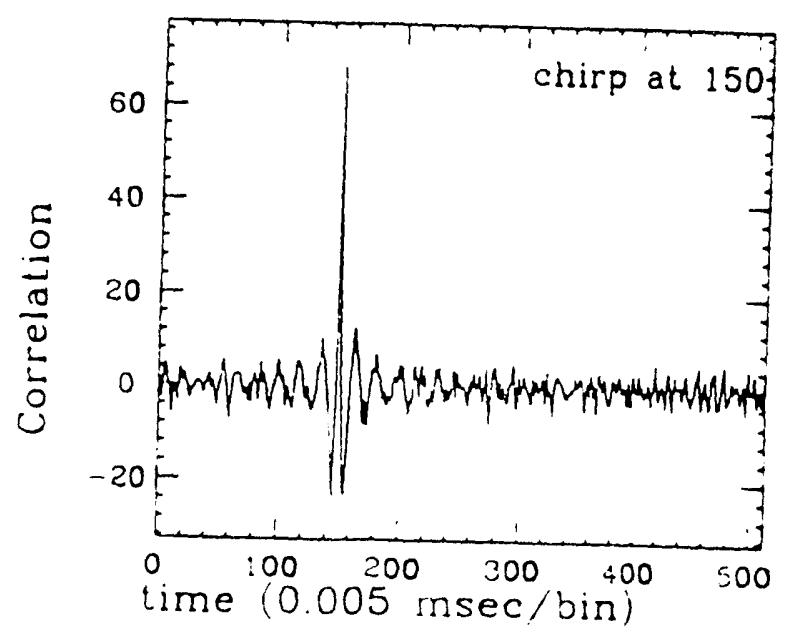

1-9. a) Noise-free constant-frequency ping. b) Constant-frequency ping with superimposed white noise and amplifier clipping: c) signal extracted from noise using convolution techniques. Sote the broad correlaion peak. d) Frequency modulated ping (chirp); e) chirp with noise: f) chirp signal extracted by replica correlation technique. Note sharpness of peak. providing improved timing resolution. 


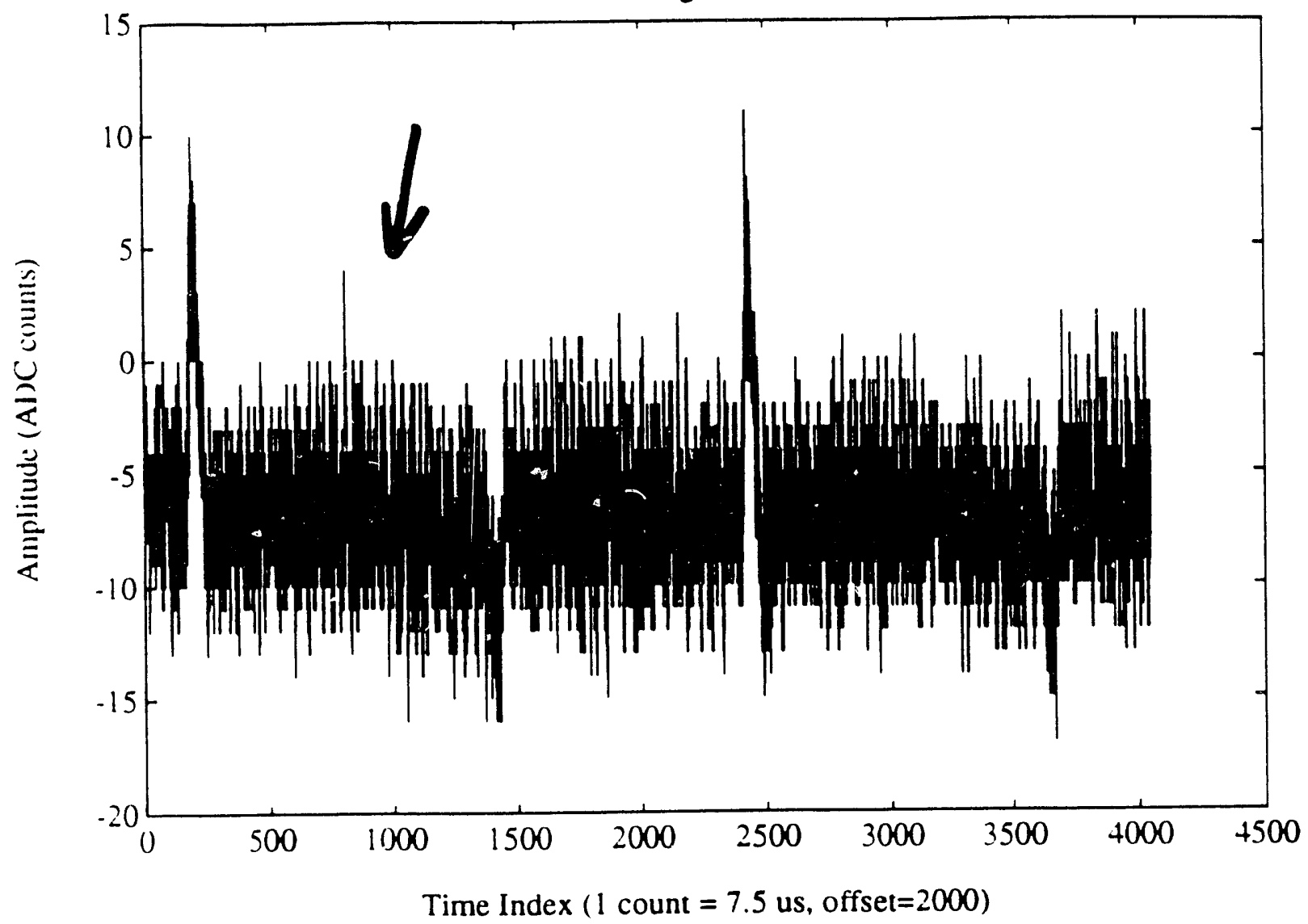

Correlation of Received Signal and Replica

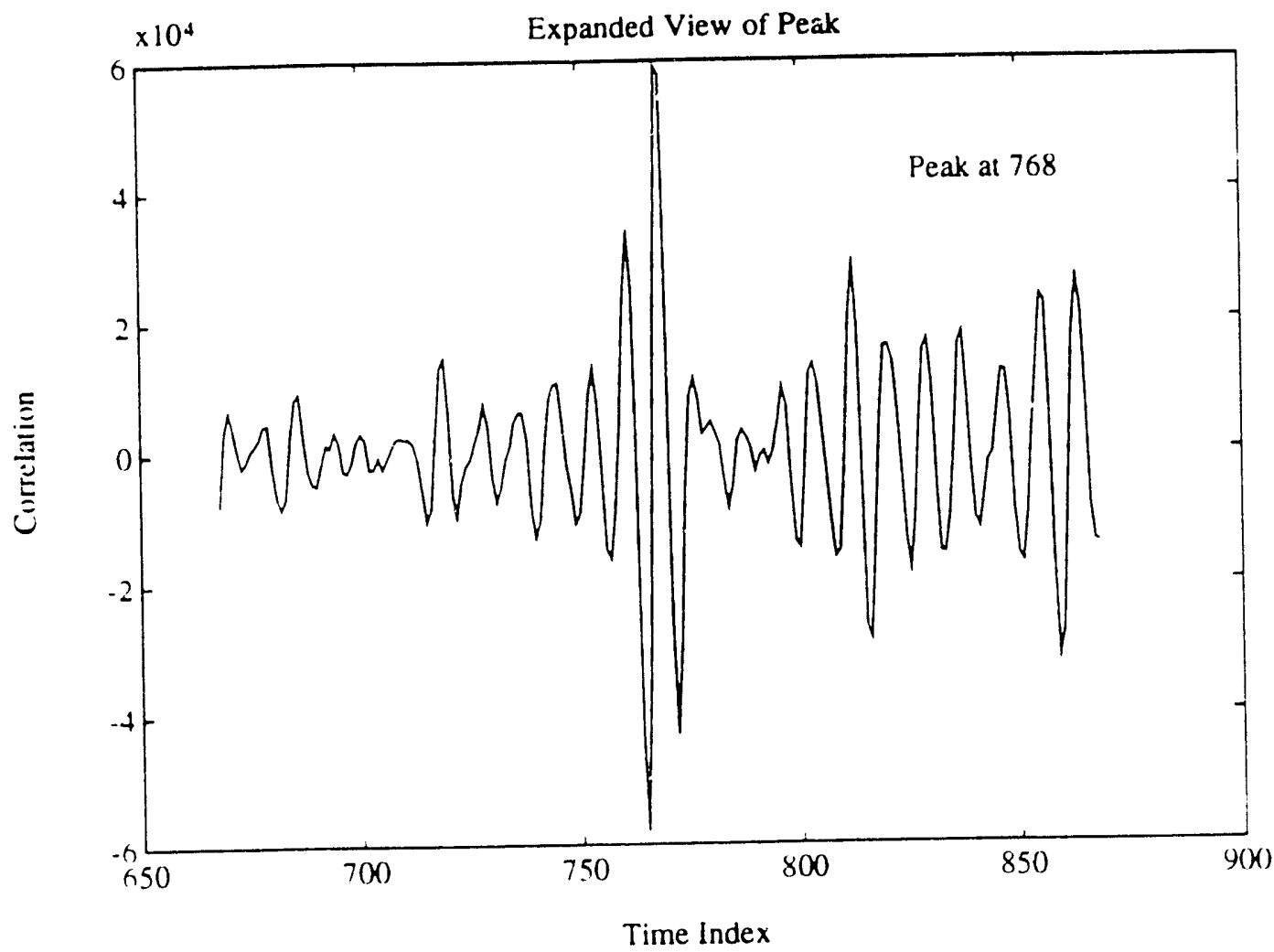

1-10) Results from near-surface, fresh-water test of positioning using $100 \mathrm{ft}$ baseline between projector and hydrophone. a) Received signal, dominated by ambient noise from nearby boats, etc: b) extracted timing signal. Timing resolution corresponds to approximately $2 \mathrm{~cm}$. 


\section{JACEE: ULTRA HIGH ENERGY NUCLEAR INTERACTIONS}

\section{A. INTRODUCTION}

JACEE (Japanese American Cosmic ray Emulsion chamber Experiment) is a US-Japanese collaboration which has performed a series of long duration balloon flight (LDBF) experiments over the past 10 years. The purpose of the experiment is to study the interactions, spectra, and composition of primary cosmic rays at energies $>1 \mathrm{TeV}$ per nucleon ${ }^{1}$. Specific scientific objectives are: a) to study multiparticle production processes at energies higher than those of proposed accelerators, using ballcon-borne cosmic ray detectors; b) to determine whether new modes of particle production are opened at extremely high nuclear densities and temperatures ${ }^{2,3}$; c) to interrelate our observations with data on extensive air showers (EAS), which are at present our only source of information on these phenomena at extreme energies; and d) to provide theoretical particle astrophysicists with essential data on cosmic ray nucleus fluxes in the $10^{13-15} \mathrm{eV}$ range, overlapping the sensitive ranges for existing ground-based ${ }^{4}$ and space-borne detectors. The experiment uses emulsion chamber detectors exposed at altitudes corresponding to 3-5 grams $/ \mathrm{cm}^{2}$ residual atmospheric overburden.

The UW group has been a key component of JACEE since we participated in initially organizing the collaboration. For all but one of the balloon flights, UW constructed the gondola and flight hardware, assembled and processed the emulsion chamber contents, and provided a significant share of the analysis effort. A member of the UW group (R. J. Wilkes) is currently US spokesman for JACEE, with T. Ogata of ICRR serving as spokesman on the Japanese side. Participating scientists are listed in Appendix II-A. While our level of effort on JACEE will have to be reduced in proportion to our increasing activity on DUMAND, existing commitments to graduate students and to the JACEE collaboration will be met.

Ten long-duration flights have been successfully completed to date, including two from Australia to South America and most recently a circumpolar flight in Antarctica. These flights (Table II-1) provide JACEE with the largest total exposure factor ever achieved by a balloon flight experiment.

In 1988 we organized an extended collaboration, SCIN/MAGIC (Spectra, Composition and Interactions / Magnetic spectrometer) including the present JACEE group with additional participants in Japan. SCIN/MAGIC was included by NASA in the first-round group of projects selected for the ASTROMAG superconducting magnet facility ${ }^{5}$ for the Space Station. Recent drastic reductions in plans for the Space Station, including termination of the ASTROMAG program, may actually benefit JACEE by permitting the same research to be performed promptly via balloon flights. Preliminary discussions indicate that NASA may now be willing to support a substantial program of long-duration balloon fights, either in Antarctica or from Australia. Our Japanese colleagues have developed and are now constructing a superconducting magnet suitable for balloon flight $^{5}$, and test flights could take place in 1992 . The principal advantage of balloon flights is that they can be carried out immediately and at low cost. Long-duration balloon flights can provide exposures equivalent for our purposes to those available via space flight.

Prompt extension of our exposure program is important because additional data are needed to resolve open issues in particle astrophysics regarding the character cosmic ray sources and of hadronic interactions at extreme energies, as well as for the planning of new heavy ion accelerators which will approach the JACEE energy range. Our proposed work thus provides a direct preview of the event types which will be encountered in the new machines. Such information will be essential for realistic planning of early experiments at these facilities.

JACEE has continued to evoke considerable interest from the theoretical and experimental particle 
physics community. For example, JACEE data were used as the focal point of seminal papers by Bialas and Peschanski ${ }^{9}$ on rapidity fluctuations. These papers stimulated a flood of theoretical and experimental effort on intermittency in particle production, as described below. JACEE results are now commonly used by the particle astrophysics community as the accepted conventional reference for primary cosmic ray spectra in the $1-100 \mathrm{TeV}$ range.

\section{B. PHYSICS MOTIVATION AND BACKGROUND}

\section{PARTICLE ASTROPHYSICS}

The cosmic ray flux at the top of the atmosphere appears to contain nuclei of all elements found on Earth, with energies up to at least $10^{20} \mathrm{eV}$ per particle. Pervading the galaxy, cosmic rays are a probe of high energy processes; their origin and accleration is intimately linked to the origin of the elements and their propagation and confinement depend on the structure of the galaxy and its magnetic fields. In the energy region around $10^{15} \mathrm{eV}$ they may carry information about compact sources which emit intense $\mathrm{fluxes}$ of $\mathrm{X}$ rays, photons, and neutrinos.

Of special interest is measurement of the elemental composition and energy spectra around $10^{14-15} \mathrm{eV}$ in total energy. This "knee" region (Fig. II-1), where observed energy spectra appear to undergo a chasge slope, has been a source of intense interest for decades, but only recently has it been possible to obtain direct measurements of spectra in this range from a variety of competing detector types.

Measurements at lower energies, $1-10 \mathrm{GeV} /$ nucleon, show that the cosmic ray composition generally follows the solar system abundances, although there are some striking differences ${ }^{7}$. For example, the secondary nuclei which result from the fragmentation of the primary (source) nuclei during traversal of the interstellar medium, are considerably more abundant than in solar system materials, but are relatively less abundant at higher energies ${ }^{8-12}$. Thus, the confinement and propagation of particles in the galaxy is energy dependent, and can be investigated directly by measuring the secondary to primary ratios as a function of energy ${ }^{13}$.

The relative abundances of the primary elements in the cosmic rays reflect the composition of the galactic material from which the cosmic rays were accelerated. The mean age of the cosmic rays is $10^{7}$ years making the cosmic rays a "young" sample of galactic matter. Comparison with the much older solar system composition shows distinct differences, notably in the $\mathrm{H} / \mathrm{He}$ and $\mathrm{C} / \mathrm{O}$ ratios. Whether these differences have a nucleosynthetic origin, are a result of the acceleration process or are due to a source effect remains an unresolved question.

Few measurements have been made of the spectra of primary cosmic rays at $100-1000 \mathrm{GeV} /$ nucleon $^{13-16}$, where the composition should become similar to that of the source. Data from previous JACEE balloon flight experiments are the most extensive source of information in this energy range, and in fact the JACEE proton spectrum has become the standard reference measurement in the field. Our data show some differences from a uniform primary index of -2.7 , which if extrapolated, would produce quite different composition at $10^{15}$ $\mathrm{eV}$ than at $10^{12} \mathrm{eV}$. JACEE data ${ }^{10,17}$ show primary spectra of $\mathrm{H}, \mathrm{He}, \mathrm{CNO}, \mathrm{Ne} \mathrm{Mg} \mathrm{Si}$, and the Fe groups consistent with a spectral index (logarithmic slope) of -2.7 up to the region of $10^{14} \mathrm{eV}$, results which need verification at higher energies and with much better statistics.

Air shower observations by a variety of methods have provided most of the data above $10^{14} \mathrm{eV}$ shown in Fig. II-1 (for details see refs. 19,20). There is a clear change of measured spectral index from about -2.7 to -3.1 around $10^{15} \mathrm{eV}$ per particle. So far only the JACEE Experiment ${ }^{17.18}$ has provided any direct 
composition measurement, using long duration balloon flight exposures of emulsion chambers of the type described here.

In addition to the primary composition, the study of the nucleus-nucleus interaction characteristics, described in the next section, will provide specific interaction data for the development of air shower cascad: models in a region well overlapping the energy range of large-scale ground level particle astrophysics experiments.

\section{HADRONIC INTERACTIONS IN NUCLEI}

Studies of central proton and heavy ion collisions allow us to examine the properties of hadronic matter under extreme conditions of high temperature and compression, which may produce a phase transition to the quark gluon plasma (QGP) state. While existence of $Q G P$ is unavoidable in conventional $Q C D$, no unambiguous evidence for observation of such a transition has yet been offered, although suggestive data from JACEE was a fundamental stimulus for extensive theoretical and experimental work in this area ${ }^{21,22}$.

According to Bjorken, McLerran, Halzen, Gyulassy and ct.hers ${ }^{22,23}$, a quark gluon plasma may be created in relativistic heavy ion collisions, perhaps even at relatively low densities, if the elementary hadrons are composed of free constituents within the strong coupling volume of a bag. If it exists, hot quark matter should be observable by studying the central collisions of heavy ions at energy densities above approximately $1 \mathrm{GeV} / \mathrm{fm}^{3}$.

However, the energy regime above $200 \mathrm{~A} \mathrm{GeV}$ is at present arcessible only by cosmic ray studies, and this situation will exist until at least 1998, when RHIC may be orerational. The CERN SPS has accelerated sulfur nuclei $(A=32)$ to energies up to $200 \mathrm{~A} \mathrm{GeV}$. Facilities to accelerate $\mathrm{Pb}$ ions $(\mathrm{A}=207)$ at $\mathrm{CERN}$ are planned for 1993. The Brookhaven AGS has accelerated ions up to $\mathrm{Si}(\mathrm{A}=28)$ to $15 \mathrm{~A} \mathrm{GeV}$, and a facility to accelerate $10 \mathrm{~A} \mathrm{GeV} \mathrm{Au}$ ions is planned for 1992 . No facility has yet been proposed to provide energies above $\sim 1 \mathrm{TeV} /$ nucleon which will probably be needed to exceed predicted energy density thresholds for transition to the quark gluon plasma state. Even if the phase transition boundary is not reached, the copious particle production observed in central heavy nucleus collisions permits application of new techniques for exploring the properties of the hadronic interaction. For example, particle correlations and factorial moment analyses of rapidity density fluctuationz give insight into the chaotic character of the particle production process.

One possible signature for the quark matter phase transition may be a break in the $\left\langle p_{t}\right\rangle$ vs $\langle d N / d y\rangle$ distribution ${ }^{1}$, as indicated schematically in Fig. II-2a. Figure II-2b, showing just such a distribution for a sample of JACEE events, shows a striking similarity to the predicted behavior. Another signature might be copious production of low- $\mathrm{p}_{t}$ photons ${ }^{2}$. Such events are readily identifiable in JACEE detectors.

At present, no experiment has provided unambiguous evidence of of a QGP phase transition. Theoretical estimates based on conventional QCD place the expected energy density threshold in the range $0.5 \sim 2$ $\mathrm{GeV} / \mathrm{fm}^{3}$, depending upon the assumptions used. Methods for estimating the actual energy density achieved in a given event are subject to some controversy, and it is possible that central collisions of the highest mass, highest energy nuclei thus far available at accelerators (200 A GeV sulfur) produced energy densities well below threshold. JACEE data involving heavier, higher energy projectiles are subject to statistical limitations, but suggestive events have been observed. Nonetheless, the available data can provide significant new information on the conventional hadroproduction process.

The emulsion chamber technique permits measurement of pseudorapidities with precision on the order of 0.01 unit, a degree of accuracy unmatched by any other type of experiment (including conventional emulsion pellicle experiments). This, coupled with the high statistical weight of individual high multiplicity events, permits correlations studies which are impractical at lower energy densities. 
Białas and Peschanski published a paper ${ }^{6}$ on the analysis of fluctuations in pseudorapidity distributions which has stimulated extensive theoretical and experimental work aimed at investigating the chaotic character of particle production. Their seminal paper used JACEE data as an example of the utility of the proposed analysis procedure (Fig. II-3a); indeed, JACEE events at the time provided the only individual interactions with sufficient statistical weight to be useful. Fig. II-3b shows the results of applying the scaled factorial moment analysis proposed by Bialas and Peschanski to high multiplicity $\mathrm{p}+\mathrm{Pb}$ events from JACEE, in comparison with accelerator data and simulated events from the Lund monte carlo FRITIOF. This subject is discussed in more detail in Section III.

Fig II-4a shows the results of a Bose-Einstein correlations analysis of JACEE data ${ }^{24}$, indicating that the hadronic source region has dimensions as expected from simple geometrical superposition pictures; i.e., the source region has the diamter of the projectile. On the other hand, Fig. II-4b shows an example of an event which displays an anomalously high ratio of photons to charged secondaries. Normal hadroproduction yields approximately equal numbers of charged particles and photons (i.e., $\pi^{+1-}: \pi^{0}=2: 1$ ). Detection of events of this type within the relatively small sample size of JACEE may suggest new interaction features at extremely high energies.

\section{EXPERIMENTAL TECHNIQUE}

A complete description of the experimental techniques used in JACEE has been published ${ }^{25}$, so we will provide only a brief summary here. A typical ballon flight emulsion chamber, shown in Figure II-3 (the chamber structure used in our recent Antarctic tlight), consists of double-coated nuclear emulsion plates, Xray films, etchable plastic sheets (CR39) and $\mathrm{Pb}$ sheets 1.0 and $2.5 \mathrm{~mm}$ thick. The chamber is segmented into sections which provide primary charge identification, target materials, and energy estimation via ionization calorimetry. All components are precision-machined to fit into an alignment box which permits spatial relationships between components to be known to better than $100 \mu \mathrm{m}$.

The detector satisfies four basic requirements for the proposed experiment: (1) large geometrical factor, (2) accurat: charge determination, with charge resolution essentially independent of energy, (3) reliable energy measurement, with energy resolution independent of (and to some extent, improving with) energy; (4) extremeiy precise angular resolution ( $\leq 0.1 \mathrm{mrad})$ for secondary charged particles.

Charges of primary particles are determined by combining grain-count and delta-ray count data from the emulsion plates with the CR39 data. Charge resolution for iron is $\sim 2.0$ units, with better resolution for lighter nuclei.

The energies of the primaries can be determined by a combination of techniques. The minimum path length through the $\mathrm{Pb}$ of the calorimeter section typically provides at least 6 radiation lengths for development of electromagnetic cascades. Ionization can be determined by counting the number of tracks within a given radius at several depths. The energy resolution improves with energy from about $20 \%$ at $0.3 \mathrm{TeV}$. The energy going into minimum ionizing charged tracks can be independently estimated by assuming $p_{t} \sim<p_{t}>\sim 0.4$ $\mathrm{GeV}$; thus $E \sim 0.4 \csc (\theta)$. Similarly, the energies of heavy fragments can be estimated from their polar angles by well established techniques ${ }^{20}$. The average overall accuracy of primary energy measurement in this experiment is calculated to be better than $35 \%$, roughly independent of energy. As indicated, we expect to perform a series of flights with a hybrid super-conducting magnet system, which will provide direct momentum measurements for secondary tracks.

Tracing of secondary tracks to the event vertex, and tracing the primary track to its entry point, have in 
past been the most time consuming aspect of emulsion chamber analysis. However, JACEE data acquisition rates have shown steady improvement, made possible by refinements in emulsion chamber analysis procedures and equipment. Our group has developed a Computer-Aided Tracing and Measuring system (CAT/CAM) which greatly increases data reduction rates. (This system is described in more detail in Section III). As data accumulates, the energy range of interest can be pushed up, resulting in fewer events per stack to be traced and analyzed. On the other hand, the complexity of the individual events increases with energy. Thus the data reduction effort is diminished while the analysis time per event increases.

The high spatial resolution of emulsions imparts significant advantages to this experiment. It allows studying multiplicity, rapidity distributions, spatial correlations of particles etc. at produced multiplicities that would saturate other track visualizing techniques. Production angles of charged tracks can be determined to an accuracy on the order of tenths of a milliradian (i.e., significant measurements can be made up to 14 units in pseudorapidity). The low density of the target section means that measurements can be made in several successive layers with negligible interference from secondary interactions and photon conversions. For the photons, true rapidity can be computed using the measured energy and the production angle determined by measuring the cascade starting point coordinates. About half of the interactions recorded occur in the calorimeter section, due to the chamber mass distribution.

\section{PROGRESS TO DATE}

\section{Long Duration Balloon Flights}

The JACEE collaboration has performed ten successful balloon flights to date (see Table II-1), including two intercontinental long-duration flights, launched in Alice Springs, Australia and recovered in South America after $120 \sim 140$ hours, and the JACEE-10 circumpolar flight in Antarctica, lasting 205 hours. These flights more than double our total exposure factor, and allow us to probe a significantly higher energy regime.

Our students and technicians in Seattle built an automatic onboard digital flight data recorder which provides a continuous record of altitude and package temperature, and is used to "turn off" the detector during temporary altitude sags by shifting a detector layer out of registration with the main chamber.

\section{Superconducting Magnetic Spectrometer}

As mentioned previously, JACEE has reached agreement with ICR, ISAS and KEK in Japan to use a large superconducting magnet for a hybrid detector. The SCIN/MAGIC experiment will provide momentum and charge data for particles in the central rapidity region. The magnet was originally designed as a prototype for the space station ASTROMAG project. It will be ideal for balloon flights due to its low weight and self-contained cryogenic system. The magnet will provide a field of nearly $2 \mathrm{~T}$ over an area large enough to accomodate an oversized JACEE emulsion chamber (Fig. II-6a). The MAGIC detector, a modified emulsion chamber with minimal target mass and no calorimeter section, allows tracking of individual charged particles from the interaction vertex with spatial resolution of 1 micron. The resultant maximum measurable momentum for 2 Tesla will be $600 \mathrm{GeV} / \mathrm{c}$, which is sufficient for most tracks in the central rapidity region and in the target fragmentation region of interactions with $10 \mathrm{TeV}$ /amu primary energy.

Construction should be complete by the end of 1992, and a test flight in Texas is planned. Our group is developing conceptual designs for the gondola and associated electronics needed to fly SCIN/MAGIC (Fig. II-6b). The SCIN/MAGIC project will be very similar to JACEE, with NASA covering costs specifically associated with the ballon flight hardware development and flight operations, while participating institutions 
are expected to provide operating budgets for personnel and travel.

\section{Project Coordination and Data Analysis Tasks}

During the past year, new results have been published on the spectra and composition of primary cosmic rays. These data have important implications for particle astrophysics as well as particle physics, since the observed spectra reflect interaction processes in the source environment during acceleration and the interstellar medium during propagation. Copies of papers published during the past year are included in Appendix I. Results from recent flights are currently under analysis in our laboratory. During the past contract period we have considerably increased our data reduction capabilities in Seattle through acquisition of a computer aided microscope system. Hardware was obtained on lown from LBL, and extremely effective software has been developed by our students.

Preliminary reports of JACEE results have excited substantial demand for more data from the theoretical community. Subsequent flights will be directed to the study of the few very high energy, heavy nucleus events recorded in each chamber. Thus the analysis workload will be reduced, especially with the use of $x$-ray film densitometry at the earliest stages to preselect events of interest.

Recent rcorganization of emulsion chamber groups in Japan will include collaborators from the Institute of Space and Aeronautical Science (ISAS), the Japanese equivalent of NASA, in our future activities. This arrangement is most welcome as our group has a long-standing research relationship with the present director of ISAS, J. Nishimura. Results from our cooperative emulsion chamber experiment with Nishimura's group ${ }^{27}$ still represent the best available data on the primary cosmic ray electron spectrum, a sensitive indicator of galactic cosmic ray propagation parameters. Emulsion chambers exposed to detect ultra-high energy nucleus interactions can be readily analyzed to extend the electron spectrum datatase. The addition of ISAS expertise will provide a most productive cooperative program. 


\section{REFERENCES for Section II:}

1. W. V. Jones, Ann. Rev. of Nucl. and Part. Sci., 3771 (1987).

2. L. Van Hove, Phys. Lett. 118B, 138 (1982).

A. Bialas and W. Czyz, Inst. of Nuclear Physics (Krakow) preprint, 1983.

3. F. Halzen and H. Liu, Phys. Rev. Ds2, 1716 (1985).

4. H. Krimm, et al, Proc. Workshop on High Energy Gamma Ray Astronomy, p. 122, J. Matthews, ed., AIP, New York, 1991; R. Ellsworth, loc. cit., p. 99.

5. A. Makida, et al, Proc. Int. Conf on Magnet Techn., KEK preprint KEK-89-147.

6. A. Białas and R. Peschanski, Nuclear Physics B273 703 (1986); ibid., Bs08 857 (1988).

7. J. A. Simpson, Ann. Rev. Nucl. Part. Sci., 98, 323 (1983).

8. L. H. Smith, A. Buffington, G. F. Smooth, L. W. Alvarez, and M. A.Wahlig, Astrophys. J., 180, 987 (1973).

9. W. R. Webber, J. A. Lezniak, and J. Kish, 13th ICRC, Conference Papers 1, p. 248 (1973).

10. E. Juliusson, Astrophys. J., 191, 331 (1974).

11. C. D. Orth, A. buffington, G. F. Smoot, and T. S. Mast, Astrophys. J., 226, 1147 (1978).

12. V. K. Balasubrahmanian and J. F. Ormes, Astrophys. J., 186, 109 (1973).

13. M. Garcia-Munoz, J. A. Simpson, T. G. Gusik, J. P. Wefel and S. H. Margolis, Astrophys. J. Suppl., 64, 269 (1987).

14. (a) M. Ryan, J. F. Ormes, and V. K. Balasubrahmanyan, Phys. Rev. Lett., 28,985 (1972) and erratum op cit., 1497 (1972), (b) M. Simon et al. Astrophys. J. 239, 712 (1980).

15. W. R. Binns, M. M. Israel, J. Klarmann, E. C. Stone, C. J. Waddington, et al., Astrophys. J., s24, 1106 (1988).

16. J. M. Grunsfeld, J. L'Heureux, P. Meyer, D., and S. P. Swordy, Astrophys. J. Letteri, 327, L31 (1988).

17. T. H. Burnett, et al, Phys. Rev. Lett., 51, 1010 (1983).

18. T. H. Burnett et al., (JACEE Collaboration), Proc. Symposium on Cosmic Abundances of Matter, Univ. of Minnesota (1988) (in press).

19. A. A. Watson, Proc. 19th ICRC, 9, 111 (1985).

20. C. E. Fichtel and J. Linsley, Ap. J., 300, 474, 1968.

21. A. Goldhaber and H. Heckman, Ann. Rev. Nucl. Sci. 28, 161 (1978).

22. J. D. Bjorken, Physical Review D27, 140 (1983).

L. McLerran, Physics Reports 88, 379 (1982).

F. Halzen and C.M. Liu, Physical Review D25, 1842 (1982).

M. Gyulassy, et al., Nucl. Phys. B2s7, 477 (1984).

23. L. McLerran, Rev. Mod. Phys. 58, 1021 (1986) and references therein.

F. Halzen, Contemp. Phys. 24, 591 (1983).

M. Gyulassy, in "Quark Gluon Plasma”, R. Hwa, ed., World Scientific, Singapore, 1990.

24. T. H. Burnett, et al, Proc. XXI Int. Cosmic Ray Conf., 8116 (1990); S. C. Strausz, unpublished thesis, Univ. of Wash., 1989.

25. T.H. Burnett, et al, Nuclear Instr. and Methods A251,583 (1986).

26. S.C. Strausz et al., Nucl. Tracks 7, 79 (1983).

27. J. Nishimura et al, Ap. J. 238, 394 (1980).

T. Saito, J. Phys. Soc. Japan 30, 1535 (1971). 
Table II-1: JACEE BALLOON FLIGHTS

\begin{tabular}{|c|c|c|c|c|c|}
\hline Flight & $\begin{array}{l}\text { Launch } \\
\text { Date }\end{array}$ & $\begin{array}{l}\text { Launch } \\
\text { Site }\end{array}$ & $\begin{array}{l}\text { Altitude } \\
\left(\mathrm{g} / \mathrm{cm}^{2}\right)\end{array}$ & $\begin{array}{c}\text { Duration } \\
\text { (hours) }\end{array}$ & $\begin{array}{l}\text { Unit (Area/Unit) } \\
\qquad\left(\mathrm{cm}^{2}\right)\end{array}$ \\
\hline JACEE-O & $5 / 79$ & $\begin{array}{l}\text { Sanriku, } \\
\text { Japan }\end{array}$ & 8.0 & 29.0 & $1(40 \times 50)$ \\
\hline JACEE-1 & $9 / 79$ & $\begin{array}{l}\text { Palestine, } \\
\text { Texas }\end{array}$ & 3.7 & 25.2 & $4(40 \times 50)$ \\
\hline JACEE-2 & $10 / 80$ & $\begin{array}{l}\text { Palestine, } \\
\text { Texas }\end{array}$ & 4.0 & 29.6 & $4(40 \times 50)$ \\
\hline JACEE-3 & $6 / 82$ & $\begin{array}{l}\text { Greenville, } \\
\text { S. Carolina }\end{array}$ & 5.0 & 39.0 & $1(50 \times 50)$ \\
\hline $\mathrm{JACEE}-4$ & $9 / 83$ & $\begin{array}{l}\text { Palestine, } \\
\text { Texas }\end{array}$ & 5.0 & 59.5 & $4(40 \times 50)$ \\
\hline JACEE-5 & $10 / 84$ & $\begin{array}{l}\text { Palestine, } \\
\text { Texas }\end{array}$ & 5.0 & 15.0 & $4(40 \times 50)$ \\
\hline JACEE-6 & $5 / 86$ & $\begin{array}{l}\text { Palestine, } \\
\text { Texas }\end{array}$ & 4.0 & 30.0 & $4(40 \times 50)$ \\
\hline JACEE-7 & $1 / 87$ & $\begin{array}{l}\text { Alice Springs, } \\
\text { Australia }\end{array}$ & 5.5 & 150.0 & $3(40 \times 50)$ \\
\hline $\begin{array}{c}\text { JACEE-8 } \\
\mathrm{m}^{2}-\mathrm{hr}\end{array}$ & $2 / 88$ & $\begin{array}{l}\text { Alice Springs, } \\
\text { Australia }\end{array}$ & 5.0 & 120.0 & $3(40 \times 50)$ \\
\hline JACEE-9 & $10 / 90$ & $\begin{array}{l}\text { Ft. Sumner, } \\
\text { New Mexico }\end{array}$ & 4.0 & 44 & $4(40 \times 50)$ \\
\hline JACEE-9 & $12 / 90$ & $\begin{array}{l}\text { McMurdo Sound, } \\
\text { Antarctica }\end{array}$ & 3.5 & 204 & $2(40 \times 50)$ \\
\hline
\end{tabular}


Figures for Section II:

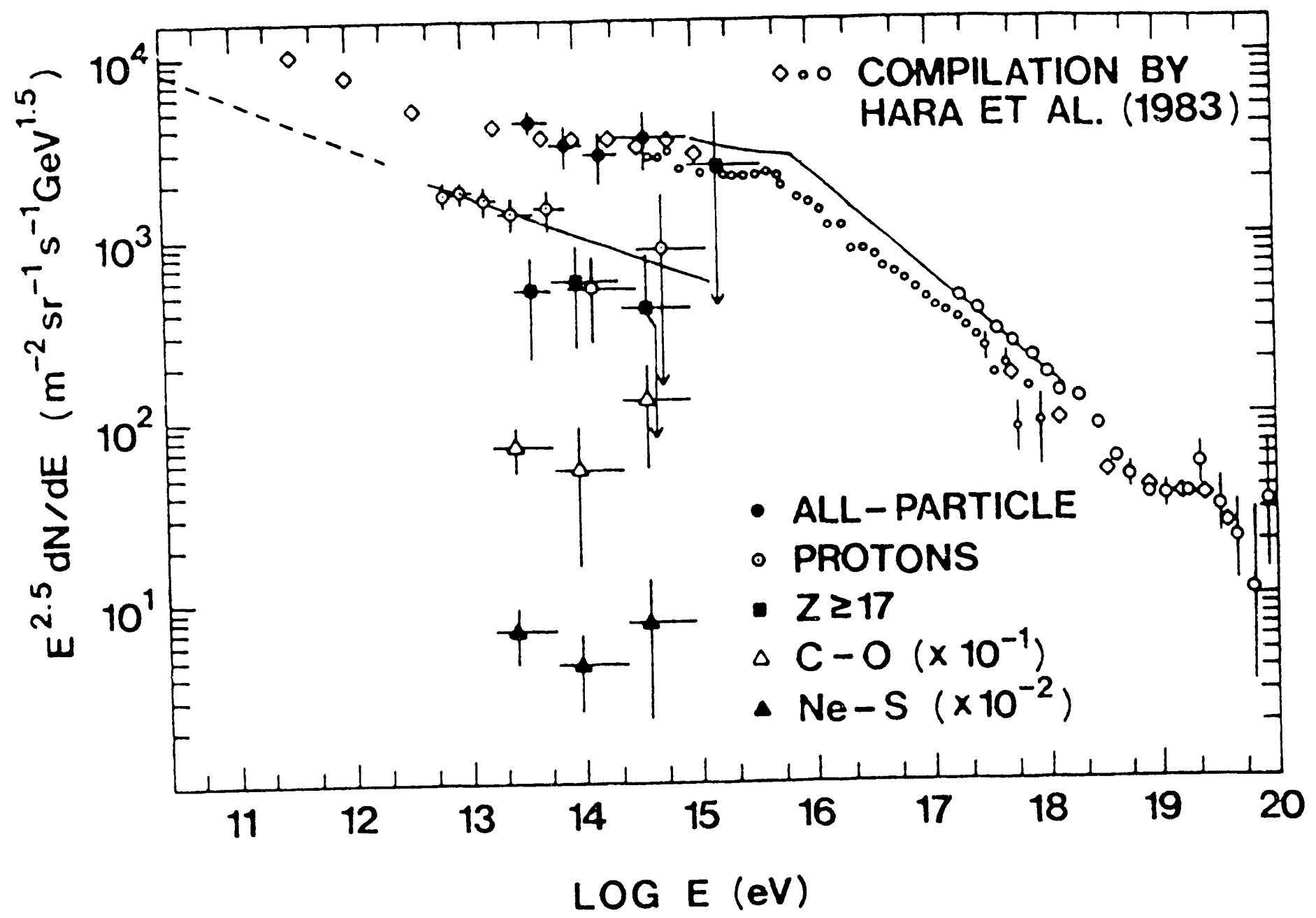

[1-1. JACEE results on primary cosmic ray spectra. showing knee region. Differentual spectra for all charged partucles. protons. alpha particles and heavier nuclei are shown in terms of total particle energy. Spectra have been scaled by the conventional factor of $E^{25}$ for clarity. 

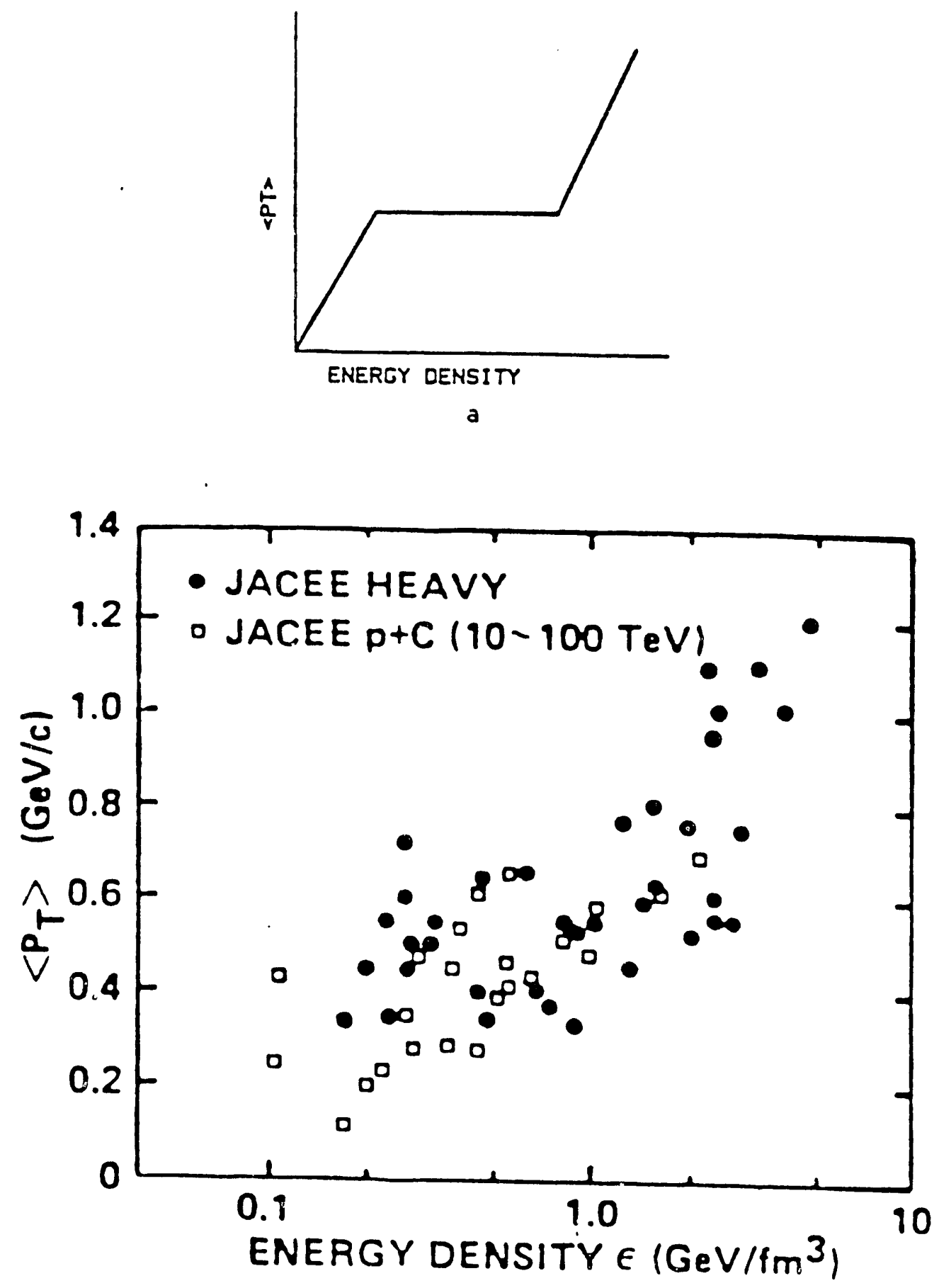

b

11-2. at Sichematic view of sirnature for phase transition in $p_{t} v \mathrm{~s} \rho$, as suggested by van Hove (ref. 2). b) JACEE results on $p_{t}$ is $\rho$. for heavy ion and proton interactions. 

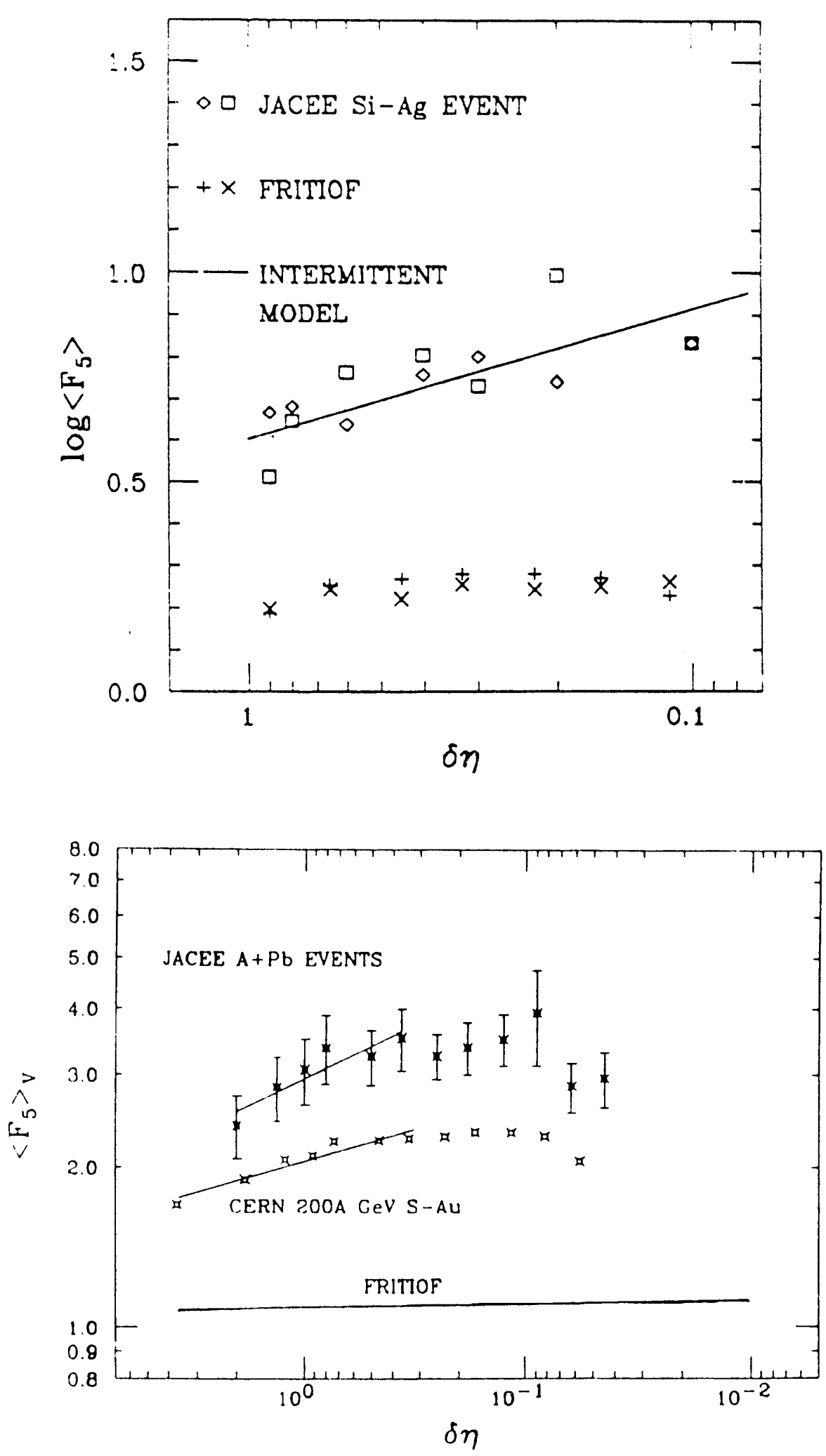

II-3. a) Scaled Factorial Moment (SFM) analysis of JACEE Si+Ag event, following Bialas and Peschanski (ref. 6). Two separate binnings of the pscudorapidity distribution are used. Results from the Alpha Model ${ }^{5}$. which includes intermittency by definition. are shown for comparison. b) Similar analysis of JACEE $\mathrm{p}+\mathrm{Pb}$ interactions: results from EMCO 01 and the Lund monte carlo FRITIOF (which contains only conventional profuction mechanisms, are shown for comparison. See Section III of this Proposal for details of intermittency studies. 

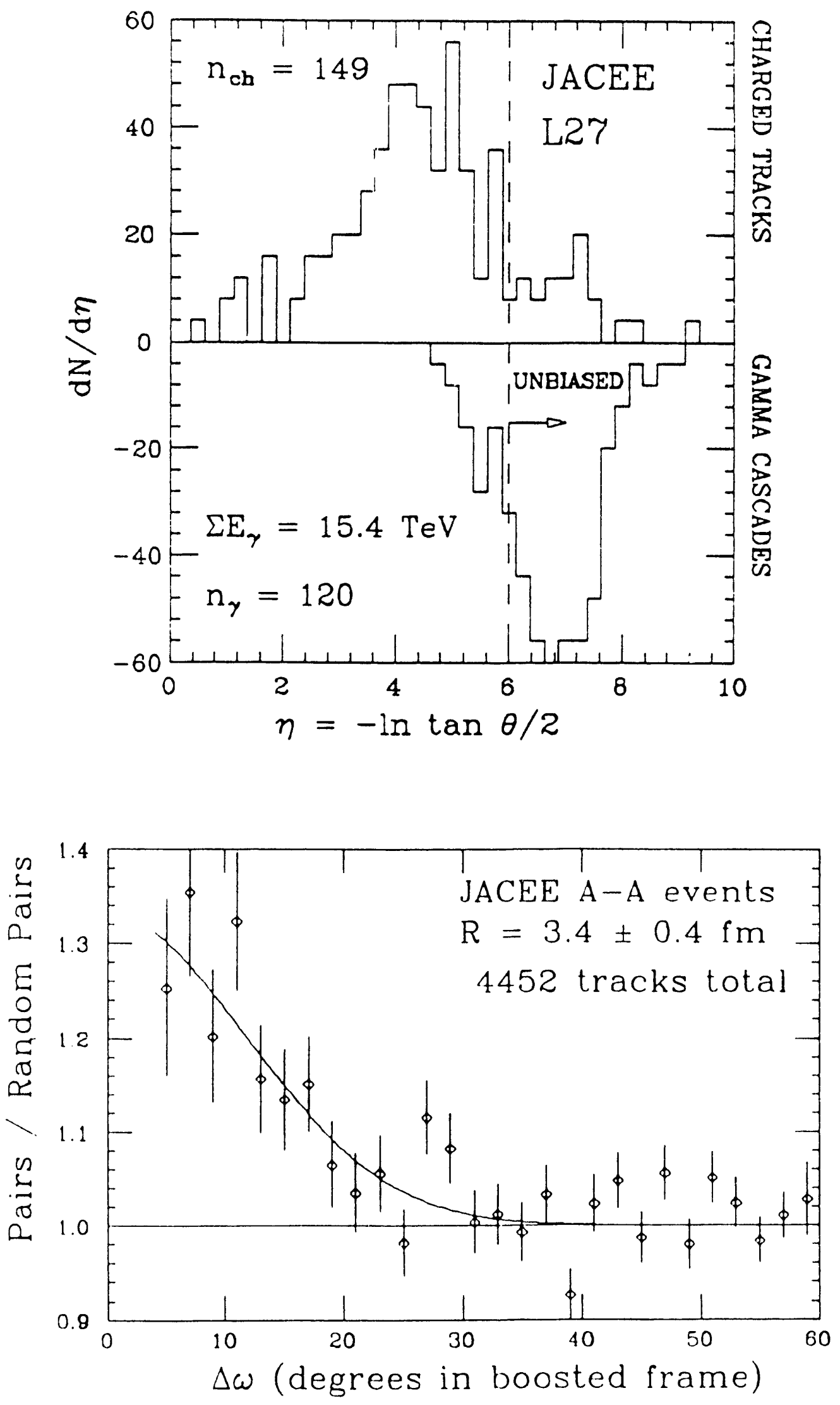

II-4. a) Example of an event with anomalous charged to neutral secondary ratio. b) Analysis of Bose Einstein orrelations made using high-resolution pseudorapidity measurements from JACEE. 


\section{JACEE-10 (Antarctica) Chamber Structure 12/90}
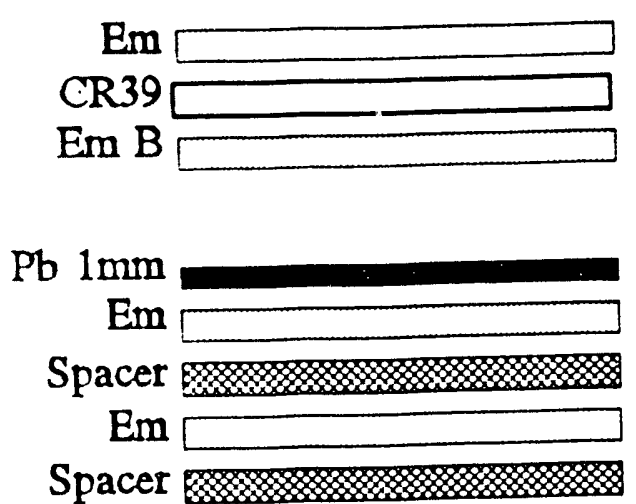

$\mathrm{Em}$

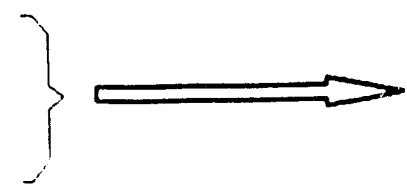

Primary Section

HRH

(cycle 2 only)

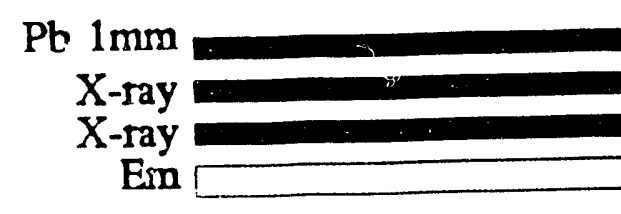

$\mathrm{Pb} 2.5 \mathrm{~mm}$

$\mathrm{X}$-ray

$X$-ray

Em
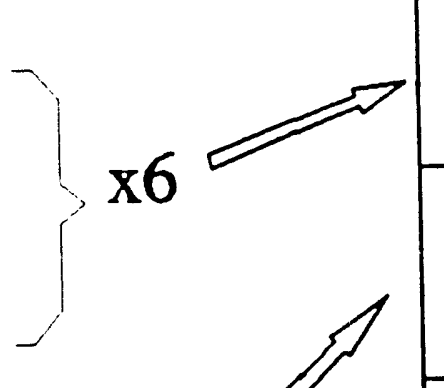

Target Section

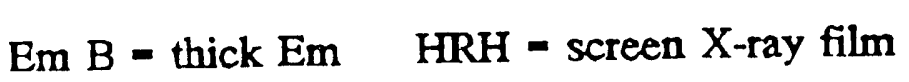



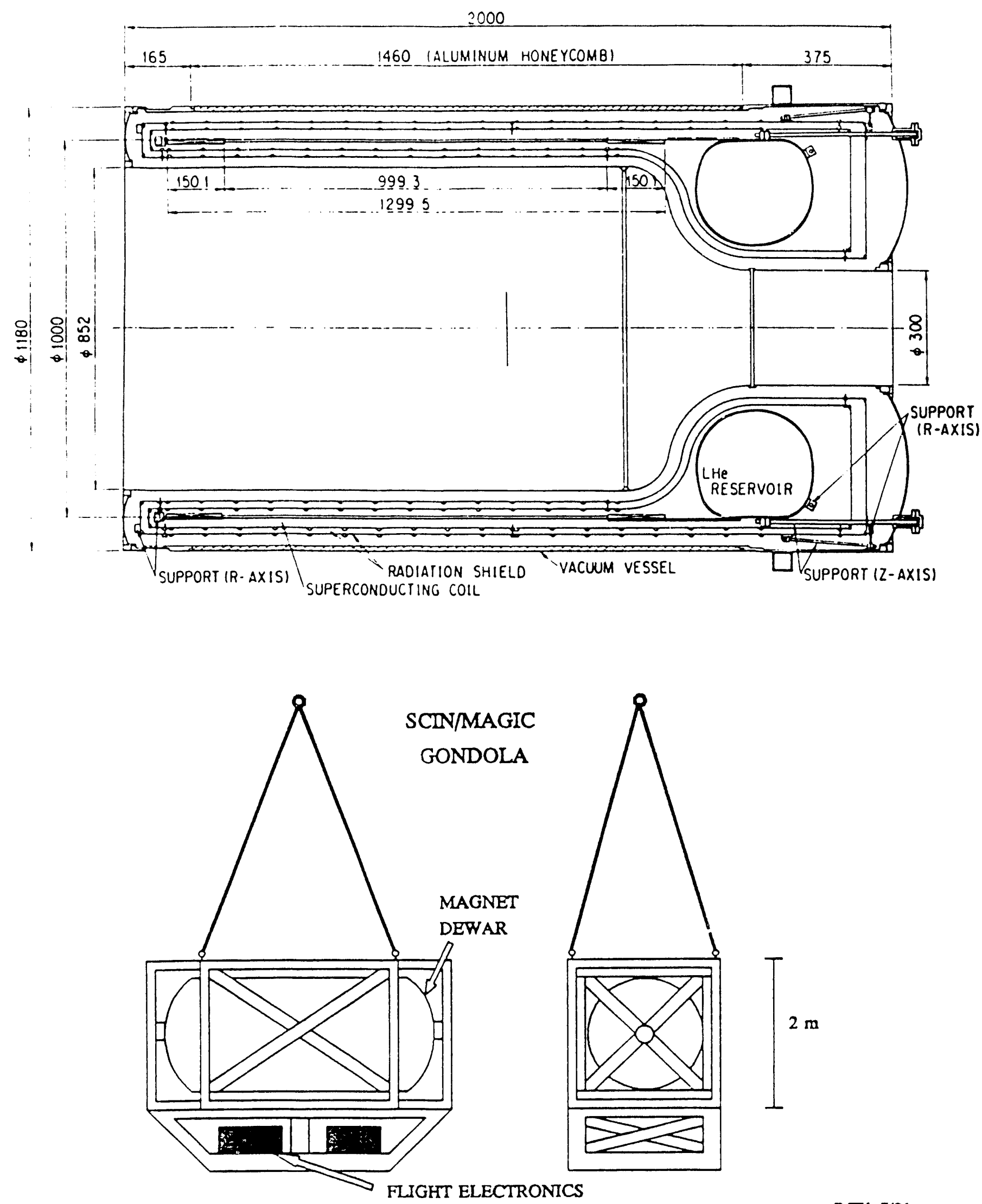

RJW, 7/91

11-6. a) SCIN/MAGIC superconducting magnet for balloon flight experiments. currenty under construction in Japan. b) Schematic design for magnet gondola to be built by UW. 


\section{ACCELERATOR INTERACTIONS STUDIES: EMU01/E815/E863/E665}

\section{A. EMU01: PHYSICS MOTIVATION AND RECENT RESULTS}

Studies of heavy ion collisions with high energy density and other high energy hadroproduction processes will enhance our understanding of the nature of the hadronic interaction. In particular, the possible existence of a quark matter phase transition, signalled by anomalies in rapidity density distributions ${ }^{1}$, strange-particle production ratios ${ }^{2}$ and $\mathrm{p}_{t}$ distributions ${ }^{3}$ is a subject of substantial current theoretical and experimental activity ${ }^{4}$. Our group is in a unique position to contribute to this area of research through our participation in JACEE as well as the complementary accelerator experiments described here.

We are members of an international collaboration which is investigating heavy-ion interactions using emulsion chambers and conventional pellicle stacks exposed to accelerator beams at CERN and BNL. Ingvar Otterlund of the University of Lund is spokesman for CERN experiment EMU01, and R. J. Wilkes of our group is spokesman for the corresponding BNL experiments, E815 (completed) and E863 (approved for exposure in 1992). The EMU01 Collaboration includes participants from Canada, China, France, Germany, India, Sweden and the USSR as well as the USA, as listed in Appendix II-C. This represents, to our knowledge, the largest and most powerful collaboration ever assembled for an emulsion experiment. Exposures have been performed at BNL and CERN, using beams of mass $A=16-32$ and energy $15-200 A \mathrm{GeV}$, with unified and consistent experimental procedures and analysis criteria. Data acquisition and analysis are proceeding rapidly and efficiently, and a substantial body of results have been published ${ }^{5}$ (Appendix I). The combination of emulsion techniques employed provides unequalled resolution for tracking and angular distributions, combined with a minimum-bias sample of events analyzed by the same group using identical criteria.

Table III-1 summarizes the EMU01 database as of the latest release. Lund University serves as the data center for the collaboration, with all groups submitting event data files in a standard format (established by the UW group). We divide the data into central, semi-central and peripheral events according to the total charge observed within a narrow cone about the beam direction: for $200 \mathrm{~A} \mathrm{GeV}$ sulfur data, central events have total charge $Z<6$ within $\theta<3 \mathrm{mrad}$, and semicentral have $Z<11$ in that range; the criteria differ slightly for other beam/energy combinations. Minimum-bias samples are obtained by along-the-track scanning in emulsion pellicles.

We have discussed the theoretical implications and possible experimental signals of QGP production in Section II above. EMU-01, along with other accelerator experiments, has observed no unambiguous evidence of events which surpass the QGP threshold. Fig. III- 1 shows the pseudorapidity $(\eta=-\ln \tan (\theta / 2))$ distributions for central collisions of $\mathrm{S}$ ions in $\mathrm{Au}$ and $\mathrm{AgBr}$ targets, where the centrality criteria are detailed below. As shown, the data are well represented by the Lund monte carlo program FRITIOF, which reproduces only conventional physics, indicating that these distributions can be explained in terms of a relatively simple superposition picture where the number of participant nucleons is the key parameter.

On the other hand, a distinctive feature of central ultrarelativistic heavy ion collisions is the appearance of significant fluctuations in rapidity density. This phenomenon has been seen in both cosmic ray experiments ${ }^{6}$ and in accelerator experiments ${ }^{7}$. It has been suggested that a quark-gluon plasma (QGP) phase transition could give rise to such behavior. One way to investigalie such fluctuations is to examine the moments of $\partial n / \partial \eta$ as a function of bin size $\delta \eta$. Current interest in this subject was stimulated by a series of papers from Bialas and Peschanski ${ }^{8}$ discussing the method of Scaled Factorial Moments (SFM) for such analyses. SFMs remove trivial effects referred to as "statistical noise" which are present when conventional 
moment analyses are applied to finite experimental data.

The behavior of the moments may reveal fundamental features of the particle production process ${ }^{0,10}$. For example the factorial moments $F_{q}$ of simulated events produced by a simple cascade production model showed a power law behavior ${ }^{8}$. Such linear behavior (on a log-log plot of SFMs vs scale size $\delta \eta$ ) is defined as "intermittency", a term borrowed from the study of chaotic turbulent fluid flow. A large number of experimental and theoretical results have been published on this subject ${ }^{11}$.

Although Bialas and Peschanski showed that individual very large events can sometimes provide adequate statistics for SFM analysis, using a JACEE event as their example, in practice it is necessary to average over many events. In the "vertical" averaging procedure, one averages bin contents over the ensemble before performing the moment analysis, so the qth factorial moment is

$$
\left\langle F_{q}\right\rangle_{V}=\frac{1}{M} \sum_{m=1}^{M} \frac{1}{N_{\text {evte }}} \sum_{i=1}^{N_{\text {ente }}} \frac{k_{m, i}\left(k_{m, i}-1\right) \ldots\left(k_{m, i}-q+1\right)}{\left\langle k_{m}\right\rangle^{q}}
$$

where $N_{\text {evt }}$ is the number of events in the sample, $k_{m . i}$ is the content of bin $m$ in event $i$, and $\left\langle k_{m}\right\rangle=$ $N_{e v t}^{-1}, \sum_{i=1}^{N_{e} \cdot t} k_{m . i}$ is the average content of bin $m$ over the ensemble of events. In the vertical analysis the moments are normalized by the average multiplicity in a given bin, so this method removes contributions caused by the overall shape of the $\eta$ distribution, i.e., variations in average bin content as a function $\eta$. Our group was among the first to emphasize the advantages of this mode of analysis ${ }^{12}$

The vertical technique was applied to a sample of 135 central $200 \mathrm{~A} \mathrm{GeV} \mathrm{S-Au} \mathrm{events.} \mathrm{The}\left\langle F_{q}\right\rangle_{V}$ exhibited a rise consistent with a power law dependence upon $\delta \eta$ for bin sizes greater than 0.4 units of $\eta$ but became independent of $\delta \eta$ for smaller bin sizes, as shown in Fig. III-2. But for an equal sample of events from FRITIOF, the $\left\langle F_{q}\right\rangle_{V}$ remained constant.

As Fig. III-2 shows, a rise consistent with a power law behavior for decreasing bin size is observed, implying that there are non-statistical fuctuations on scales greater than 0.4 units of $\eta$ which disappear at smaller scales. Because our experimental resolution is at least an order of magnitude smaller, these results indicate the absence of intermittent behavior below this scale. This rise in the moments for $\delta \eta>0.4$ represents a possible departure from the "conventional" physics embodied in the Lund Monte Carlo since it is absent from simulated events analyzed in the same way. Most other experiments cannot provide information on scales smalier than 0.1 in $\delta \eta$.

In ref. $[13 \mid$ we proposed a scaling relation for the slopes of the SFM plots:

$$
\phi_{\eta} \equiv \partial\left(\ln F_{\eta}\right) / \partial(\ln \delta \eta)=\left(\begin{array}{l}
q \\
2
\end{array}\right) \phi_{2}=\frac{q !}{2(q-2) !} \phi_{2} .
$$

using the approximation $\ln F_{q} \sim F_{q}-1$, valid when the moments are $\sim 1$. Slopes of the SFMs may indicate intermittency in particle production, and are relatively robust with respect to specific experimental cuts which may be applied. However, the validity of (1) demonstrates that most of the statistically significant information in the SFMs is already present in $F_{2}$.

SFMs are dominated by contributions from the two particle correlation distribution; factorial cumulants remove these correlations from higher order moments and explicitly demonstrate higher order correlations, if present. The second factorial cumulant for a given bin in $\eta$ is $f_{2}=\langle k(k-1)\rangle-\langle k\rangle^{2}$, where $k$ is the number of particles within the bin, with similar definitions for higher orders ${ }^{14.15}$. The factorial cumulant moments are given by 


$$
K_{q}=\frac{1}{M} \sum_{n=1}^{M} \frac{f_{q}^{(m)}}{\left(k_{m}\right)^{q}}
$$

where $f_{4}^{(m)}$ is the $q$ th cumulant for bin $m$. The factorial cumulants are related to the factorial moments ${ }^{14}$; for example, $K_{2}=F_{2}-1$. For a Poisson distribution, the factorial cumulants of all orders except the first are zero ${ }^{15}$; thus any deviation from zero for a given cumulant order indicates the presence of non-statistical correlations of that order.

Straight lines were fitted to the cumulant plots over the range $0.09<\delta \eta<2.0$, and slopes were calculated on a linear scale, i.e. $\pi_{q}=\partial K_{q} / \partial(\ln \delta \eta)$. The presence of linear behavior indicates that intermittency is observable down to scales of about $\delta \eta=0.1$. If the production mechanism is the same for all energies and projectiles studied then we would expect ${ }^{13.16}$ the cumulant moment slopes to be inversely proportional to $\rho \equiv\langle\partial \mathrm{n} / \partial \eta\rangle$. In Fig. III-3 we plot the slopes $\pi_{2}$ as a function of $\rho$ for the various event samples, as well as for some minimum bias $\mathrm{p}+$ emulsion and $\pi$ +emulsion data which were measured with essentially similar procedures. These data are all consistent with inverse- $\rho$ dependence; thus the analysis of factorial cumulant moments does not su $\mathrm{u}_{\mathrm{i}}$ gest any changes in behavior for systems ranging from $\mathrm{p}-\mathrm{p}$ to $\mathrm{S}+\mathrm{Au}$, for energies ranging from $14.6 \mathrm{~A} \mathrm{GeV}$ to $200 \mathrm{~A} \mathrm{Gev}$ and up to $3000 \mathrm{GeV}$ for protons. In particular, we see no evidence for a change of behavior which would suggest a QGP phase transformation for the larger and/or more energetic systems.

Factorial and cumulant moments are integrals of the multiparticle correlations. The two particle correlation function is defined by: $R_{2}\left(\eta_{1}, \eta_{2}\right)=\rho_{2}\left(\eta_{1}, \eta_{2}\right) / \rho\left(\eta_{1}\right) \rho\left(\eta_{2}\right)-1$. Figure III-4 shows the two particle correlation function observed for central $\mathrm{S}+\mathrm{Au}$ data. While the two-particle correlation function can be fitted with a single Gaussian, the data suggest a double-Gaussian distribution, with an additional narrow peak at small $\delta \eta$. (Note that in this context $\delta \eta$ represents the separation in $\eta$ between tracks rather than bin size.) $R_{2}$ for an equivalent sample of events created by the Lund Monte Carlo program FRITIOF ${ }^{17}$ produces an essentially flat distribution, even when gamma conversions in the Au foil target are taken into account. A two Gaussian fit to the $\mathrm{S}+\mathrm{Au}$ data produces the magnitudes and widths shown. It is possible that the narrow central peak is due to Bose-Einstein correlations ${ }^{18}$; the second Gaussian seems broader than would be expected from conventional short range correlations with a scale of $1 \sim 2$ rapidity units ${ }^{19}$. Other analysis modes for these data are being investigated, including multifractal correlations ${ }^{20}$.

\section{B. EXPERIMENTAL TECHNIQUE}

The emulsion chamber technique used in the cosmic ray work described in Sect. II of this proposal can be directly applied to accelerator experiments, with a number of advantages over both the cosmic ray experiment and competing accelerator techniques. Since collecting area is of no importance, the emulsion chambers are smaller than the cosmic ray detectors, and mechanical registration of plates can be better controlled. These chambers can easily be placed inside a high-field magnet, providing magnetic spectrometry for charged secondaries. Also, noninteracting beam tracks provide an abundant supply of local fiducials, so that all track measurements can be performed with extremely high accuracy. Nuclear emulsion provides spatial resolution at least one to two orders of magnitw" finer than other detector types, and the double-coated plate technique provides a vector (rather than a single coordinate) at each detection plane, so the problem of track following in complex high multiplicity events is greatly simplified. This is especially important when a magnetic field is used for momentum analysis; the track-following problem can become intractable for events 
with multiplicity greater than 500 observed in proportional chamber systems.

In both EMU-01 and E815, we used emulsion chambers of the type shown in Fig. III-5. A relatively thick plate, with two layers of emulsion each 250 microns thick, serves as a track-sensitive target, with some chambers including a 250 micron gold foil to provide a uniform, high- $A$ target layer. Thin plates are used to observe secondary tracks, and graded spacer layers permit efficient use of point measurements in angular fitting procedures. The chambers have transverse dimensions $10 \times 10 \mathrm{~cm}^{2}$. A $5 \times 5 \mathrm{~cm}^{2}$ version was also prepared to fit into the gap of a standard CERN beam dipole magnet. We obtained a magnetic field of $\sim 2 \mathrm{~T}$ in several chambers of similar design, adequate for momentum analysis of the secondaries up to several tens of $\mathrm{GeV}$. Finally, some chambers were exposed with a Pb-emulsion calorimeter $(2 \mathrm{~mm} \mathrm{~Pb}$ plates interleaved with emulsion plates) appended to the normal front end. These chambers permit direct comparison of accelerator results with cosmic ray emulsion chamber data on $\Sigma E_{\gamma}$, the total secondary energy fraction going into electromagnetic cascades.

Our experimental technique allows the determination of: (1) $n_{0}$, the number of fast $(\beta \geq 0.7)$ particles produced; (2) $N_{h}$, the number of heavily-ionizing particles; and (3) the pseudorapidity distribution $\eta=$ $(-\ln \tan (\theta / 2))$ for the fast particles, with precision $\sim 0.01$ unit. This high resolution in $\eta$, verified by repeated measurements of events, is a crucial difference between EMU01 and most competing experiments, including other (conventional) emulsion experiments.

As with JACEE, our group in Seattle prepared all hardware required for the exposures. Emulsion plates for E815 were prepared and developed in our darkroom in Seattle, while the EMU-01 plates were prepared and subsequently processed in a darkroom facility at CERN, by personnel from our lab and the Lund group.

Track coordinates are measured either on conventional digitized stages (emulsion-plane digitizers) or using an image-plane digitizer technique, originally developed in Lund ${ }^{21}$ and adapted by our group. The "EMUPAD" measuring system consists of a digitizing pad coupled to a microcomputer. The microscopist uses a camera lucida drawing tube attachment, which projects the digitizing pad into the microscope field of view, and the digitizer is used to mark the points to be measured. A beam splitter is mounted on the drawing tube to merge an image of the computer monitor (as well as the digitizer pad surface) into the microscope field of view. After the first plate has been measured, predicted track locations for successive layers are displayed on the monitor (and thus on the microscope image of the emulsion plate). This adds greatly to the time savings, eliminating confusion and lost measurement effort. We are presently using a system of this type for most measurements of high multiplicity events. Complete measurement of a 100 prong e rent takes about 2.5 hours with this device, compared to at least a full working day on a conventional stage.

Last year, we acquired a surplus microscope system on indefinite loan from our EMU collaborators at LBL. This system includes high quality microscope optics, 0.5 micron stage coordinate digitizers on 3 axes, and a servomotor drive system. No useful software existed for this system. Our personnel repaired damaged electronics, and wrote control software to allow this system to be used efficiently as a. Computer Aided Tracking / Measuring System (CAT/CAMS), as mentioned in Section II above (Fig. III-6). As presently arranged, the system uses track data from plates already measured to locate tracks automatically in subsequent plates, in a manner similar to that described for the EMUPAD system. The difference is that the CAMS device is an image-plane digitizer and is not limited to a single field of view. It will thus provide valuable improvements in measuring efficiency for large events encountered in high energy S-Au data ${ }^{22}$.

The emulsion chamber data provide high precision angular measurements of dense events in the forward and central region. The conventional emulsion pellicle stacks provide a complementary technique which can yield a minimum-bias sample of events by along-the-track scanning, and high precision measurement of 
projectile and target fragment charges. The size of the collaboration, and the presence of large groups in the USSR and China with experience in pellicle analysis technique, makes it possible to maintain a substantial rate of data reduction. Both sets of data are checked by the Lund data center before release, and then analyzed by the collaboration with uniform criteria and procedures. No other experimental group uses both techniques in this way. In addition to new data obtained in heavy ion runs since 1986, our database provides access to a large set of older data on hadron nucleus interactions at Fermilab and SPS energies, and events from lower energy nucleus accelerators, reduced by similar procedures.

\section{PRESENT STATUS}

As the preceding discussion indicates, the EMU01 Collaboration is a very active and highly productive project which has just reached the exciting stage of having a stable and extensive database for physics analysis. EMU01 results have been presented in a substantial body of journal articles ${ }^{5}$, with those published during the past year attached in Appendix I. Our group at UW plays a key role in this collaboration, in preparation and processing of detector modules for the experimental runs, in the analysis effort, and in the coordination of activities at BNL. For example, we are at present taking the lead in preparing a draft journal article on fluctuations analyses, including studies of intermittency, cumulant moments, and two particle correlations. Although EMU01 work will in future take second priority to our DUMAND effort, we will fulfill existing commitments to the collaboration and to graduate students, and participate in this extremely productive experiment to the extent possible.

In addition to earlier exposures at BNL and CERN in 1986-87, we had a 200*A GeV sulfur beam exposure at CERN in 1990, and some scanning and measurement effort remains to be completed. The results available from the latest database distribution are summarized in Table III-1. Data reduction and analysis has been completed on a total of $>3000$ events from all exposures. An exchange database system designed and implemented by our group has played a key role in the rapid progress and efficient analysis effort by EMU-01. The database defines a minimal set of event parameters required by our analysis goals, uses a simple text file format which can easily be handled by all members of the collaboration using IBM PC type computers, and can be transferred via BITNET or by mailing floppy disks.

We recently had scientists from our collaborating institutions in Beijing and Hunan, P. Y. Zheng and J. F. Sun, visiting our lab for 6 months under a grant from the NSF US-China program. Our proposal also requested support for travel by members of our group to work sessions and a planned collaboration meeting in China; although this portion of the grant was temporarily suspended by NSF due to recent events in China, it will be reinstated this year and we expect to send a member of our group on a 1-month reciprocal visit. Drs. Zheng and Sun made significant progress on the analysis of high resolution emulsion chamber magnetic spectrometer data, and we would like to complete this effort, which has applications in JACEE as well as EMU01.

During the coming year we expect to perform a proportionate share of the data reduction and analysis in Seattle. EMU01 submitted a proposal to BNL for exposures to the $10 \mathrm{~A} \mathrm{GeV}$ Au beam planned for commissioning in 1992. Our proposal was accepted as AGS Experiment E863; the program review committee responded very positively to results from our previous exposures (E815). Another run using $\mathrm{Pb}$ ions at energies up to $200 \mathrm{~A} \mathrm{GeV}$ at CERN is expected in 1993 . These heavier projectiles may be sufficient to push the energy density over the QGP production threshold, and will in any case p:ovide interesting results on correlations and fluctuations. We have been asked by the Collaboration to prepare and expose test chambers 
to $\mathrm{Au}$ beams at $\mathrm{LBL}$, to provide test plates for chamber design refinement before the $\mathrm{Au}$ and $\mathrm{Pb}$ runs at BNL and CERN.

\section{EB65: status and future effort}

Experiment E665 at Fermilab is an on-going experiment for the study of muon scattering with hadron detection. The experiment consists of a powerful spectrometer directed to the study of deep inelastic scatteing utilizing the world's highest energy mucn beam. A primary objective for present study is the use of hadrons to study photon-nucleon interaction structure of the nucleon and hadronization. The specific topics are '! Current jet hadronization with identified hadrons, ii) Event structure relative to the virtual photon axis, iii) Target jet fragmentation and diquark fragmentation, and iv) Exclusive vector meson production. A unique feature of this experiment has been the ability to measure the structure functions a very low values of $x(x=0.001)$. E665 is a US-European collaboration organized to investigate the problems outlined above ${ }^{1}$. A list of the members of the collaboration is atiiached in Appendix II The E665 detector system is shown in Fig. III-7. The Vertex drift chamber which was added for the 1990 run has proved to be excellent and much superior to the streamer chamber used in the first run. A new target assembly allows the interchange of any of two liquid and seven solir targets between each spill. This provides substantial control of systematics in comparing results with various targets.

The 1991 run started on July 7, 1991 and we are concentrating on higher luminosity studies of the hadrons from deep inelastic scattering for hydrogen and deuterium targets. The spectrometer allows a unique study of the event structure of deep inelastic events with a total hadronic energy in the c.m. of $\mathrm{W}=22-32 \mathrm{GeV}$. It is in this kinematic range that the first clear 3-jet event structure was observed in reactions such as the electron-positron collisions. Our results provide a new look at hard QCD processes in deep inelastic scattering and permit a unique examination of the next to leading order photon-gluon fusion process which makes a substantial contribution at $x$ values below 0.05 . The new run will allow us to obtain a good statistical sample of 2 -forward jet events. It will also allow us ts continue other significant studies of the hadronization process such as flavor correlations or baryon production mechanisms using the excellent particle identification of the E665: -ctrometer. Lastly, we will be able to provide signficant results on the inclusive muon scattering structure functions at modest $Q 2$ from $x=0.001$ to 0.2 .

Our level of participation in E665 has been reduced and for 1992 our contribution will consist of participating in data taking runs and assisting with completion of data analysis, to the extent permitted by our other other commitments. 


\section{REFERENCES for Section III:}

1. L. Van Hove, Proc. XIIY Int. Symp. on Multiparticle Dynamics-1982, p. 244, W. Kittel et al eds., World Scientific, Singapore, 1983. F. Halzen and H. Liu, Phys. Rev. D25, 1842 (1982), and Phys. Rev. Letters 48, 771 (1982).

2. J. Rafelski, Phys. Reports 88, 331 (1982).

3. L. Van Hove, Phys. Lett. 118B, 138 (1982).

4. M. Gyulassy, “Quark Gluon Plasma”, R. Hwa, ed., p. 223, World Scientific, Singapore, 1990.

5. M. I. Adamovich, et al., Phys. Lett. B201, 397 (1988);

M. I. Adamovich, et al., Phys. Lett.B223, 262 (1989;,

M. I. Adamovich, et al., Phys. Rev. Lett. 62, 2801 (1989);

M. I. Adamovich, et al., Phys. Rev.C40, 66 (1989);

M. I. Adamovich, et al., Phys. Lett. 230B, 175 (1989);

M. I. Adamovich, et al., Phys. Lett. 227B, 285 (1989);

M. I. Adamovich, et al., Mod. Phys. Lett. A5, 169 (1990);

M. I. Adamovich, et al., Phys. Lett. 234B, 180 (1990);

M. I. Adamovich, et al., Zeit. Phys. C49, 395 (1991);

M. I. Adamovich, et al., Mod. Phys. Lett. A6, 469 (1991).

See also refs. 7, 12, 13, and 20.

6. T. H. Burnett, et al, Phys. Rev. Lett. 50, 2062 (1983).

7. E. Stenlund et al, Proc. Quark Matter '88, Nuclear Physics A498, 541c (1989).

8. A. Bialas and R. Peschanski, Nucl. Phys. B273, 703 (1986); A. Białas and R. Peschanski, Nucl. Phys. B308, 857 (1988).

9. P. Carruthers, Univ. Arizoia preprint AZPH-TH/89-28, and references therein (1989).

10. I. Dremin, Mod. Phys. Lett. A13, 1333 (1988); I. Dremin, Fermilab preprint (unnumbered), 1989;

11. For recent reviews of the extensive literature, see R. Hwa, Intermittency in High Energy Collisions, F. Cooper et al, eds, p. 464, World Scientific, Singapore, 1991; and W. Kittel, Proc. XX Symp. on Multiparticle Dynamics, 1990.

12. M. Adamovich, et al., Phys. Rev. Lett. 65, 412 (1990).

13. M. Adamovich, et al., LUIP 9011 (1990) (submitted to Phys. Rev.Lett.).

14. P. Carruthers, H. Eggers and I. Sarcevic, Phys. Lett. B254, 258 (1991).

15. M. Kendall, A. Stuart and J. Ord, Kendall's Advanced Theory of Statistics Vol. 1, Oxford University Press (1987).

16. D. Seibert, Phys. Lett. B240, 215 (1990).

17. B. Nilsson-Almqvist and E. Stenlund, Comp. Phys. Comm. 43, 387 (1987).

18. W. Zajc, Proc. 4th Conf. on Intersections of Particle and Nuclear Physics, to be published, 1991; W. Zajc, Phys. Rev. 35, 3396 (1987).

19. R. J. Wilkes, et al, Proc. 4th Conf. on Intersections of Particlc and Nuclear Physics, to be published, 1991 (preprint UWSEA-PUB-91-10).

20. R. Hwa, Phys. Rev. D41 1456 (1990).

21. S. Garpman, et al., Nucl. Instr. \& Meth. A269, 134 (1988); E. Ganssauge et al, Nucl. Instr.\& Meth. A 257, 314 (1988).

22. E. Olson, et al, Proc. XXII Int. Cosmic Ray Conf., paper HE-1.2.35, to be published by Trinity College, Dublin (1991). 


\section{Table III-1: Event samples used in EMU01 analyses}

$Q_{Z D}$ is the amount of charge within $\theta_{Z D}$ upon which centrality or semi-centrality are based. $N_{\text {evto }}$ is the number of events, $\left\langle n_{s}\right\rangle$ is the average charged multiplicity for each sample. $\left\langle n_{c t r}\right\rangle$ is the average charged multiplicity within a window of two units of pseudorapidity centered on the nucleon-nucleon CM rapidity. Minimum bias samples were taken from pellicle stacks only. Detector types: $\mathrm{C}=$ emulsion chamber, $\mathrm{P}=$ pellicle stack.

\begin{tabular}{|c|c|c|c|c|c|c|c|}
\hline proj./target & detector & $\mathrm{E} / \mathrm{A}(\mathrm{GeV})$ & $\theta_{Z D}(\mathrm{mrad})$ & $Q_{Z D}$ & $N_{\text {evts }}$ & $\left\langle n_{s}\right\rangle$ & $\left\langle n_{c t r}\right\rangle$ \\
\hline $\mathrm{O}+\mathrm{Em}$ & $\mathrm{C}, \mathrm{P}$ & & & & & 51.5 & 31.2 \\
\hline $\begin{array}{l}\text { central } \\
\text { semi-central }\end{array}$ & & $\begin{array}{l}14.6 \\
14.6\end{array}$ & $\begin{array}{l}39 \\
39\end{array}$ & $\begin{array}{l}0-2 \\
3-5\end{array}$ & $\begin{array}{l}103 \\
213\end{array}$ & $\begin{array}{l}51.0 \\
33.5\end{array}$ & $\begin{array}{l}31.2 \\
19.6\end{array}$ \\
\hline $\begin{array}{l}\text { semi-central } \\
\text { min bias }\end{array}$ & & $\begin{array}{l}14.6 \\
14.6\end{array}$ & & & 692 & 24.7 & 13.4 \\
\hline central & & 60 & 10 & $0-2$ & 88 & 99.2 & 57.5 \\
\hline semi-central & & 60 & 10 & $3-5$ & 169 & 69.1 & 38.9 \\
\hline $\min$ bias & & 60 & & & 474 & 46.4 & 24.4 \\
\hline central & & 200 & 3 & $0-2$ & 180 & 170.4 & 83.3 \\
\hline semi-central & & 200 & 3 & $3-5$ & 213 & 125.5 & 60.1 \\
\hline $\min$ bias & & 200 & & & 509 & 79.2 & 36.6 \\
\hline$S+E m$ & $\mathrm{C}, \mathrm{P}$ & & & & & & \\
\hline central & & 200 & 3 & $0-5$ & 115 & 342.5 & 166.5 \\
\hline semi-central & & 200 & 3 & $6-10$ & 127 & 219.9 & 102.8 \\
\hline min bias & & 200 & & & 597 & 135.2 & 62.6 \\
\hline$S+A u$ & C & & & & & & \\
\hline central & & 200 & 3 & $0-5$ & 190 & 383.9 & 196.5 \\
\hline semi-central & & 200 & 3 & $6-10$ & 85 & 289.8 & 141.1 \\
\hline
\end{tabular}


Figures for Section III:

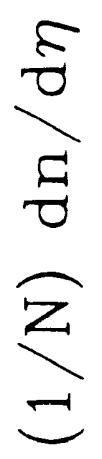

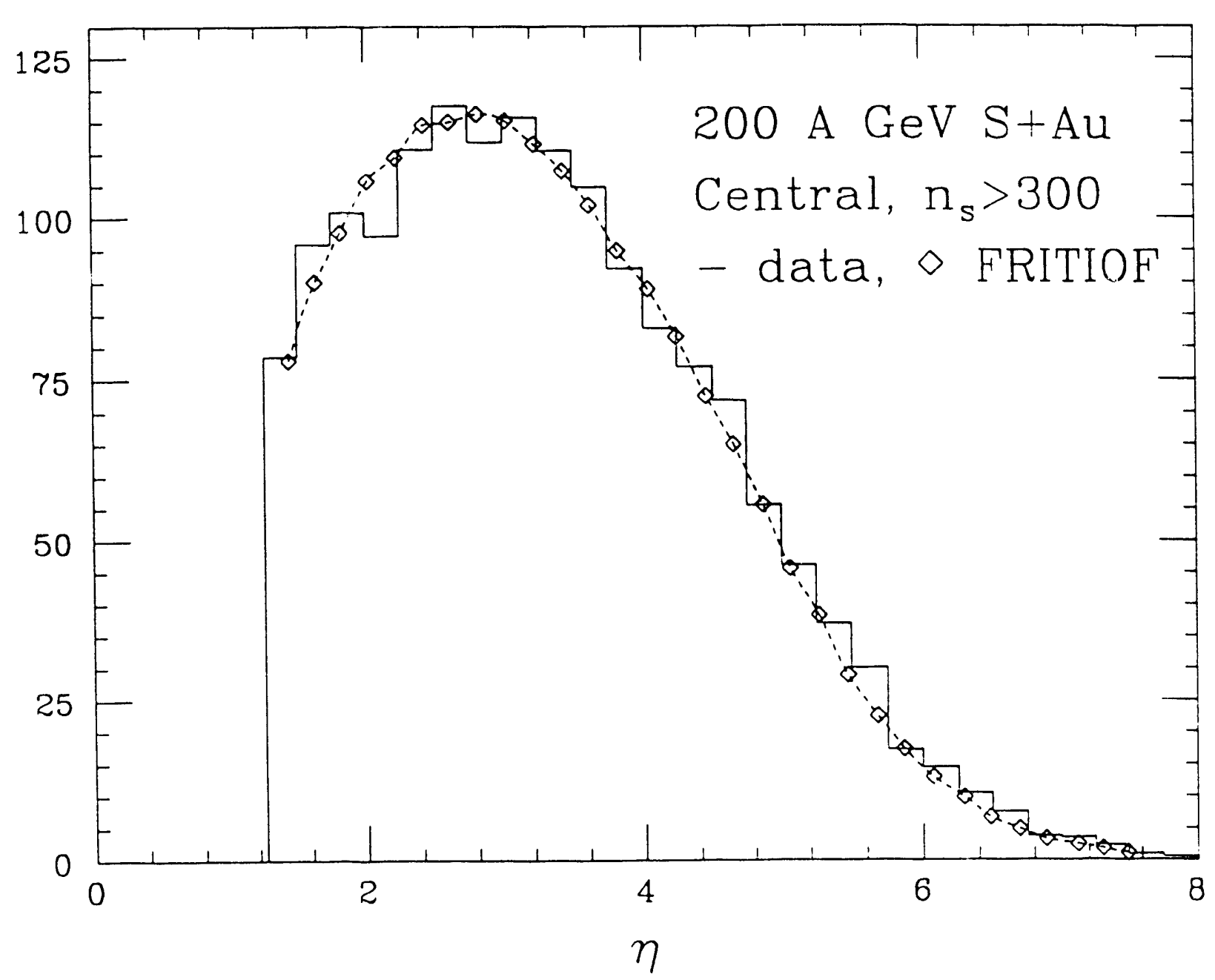

1II-1. Pseudorapidity distribution for central S + Au collisions at $200 \mathrm{~A} \mathrm{GeV}$. The FRITIOF monte carlo. effectively implementing a superposition model. provides a good representation of the data. 

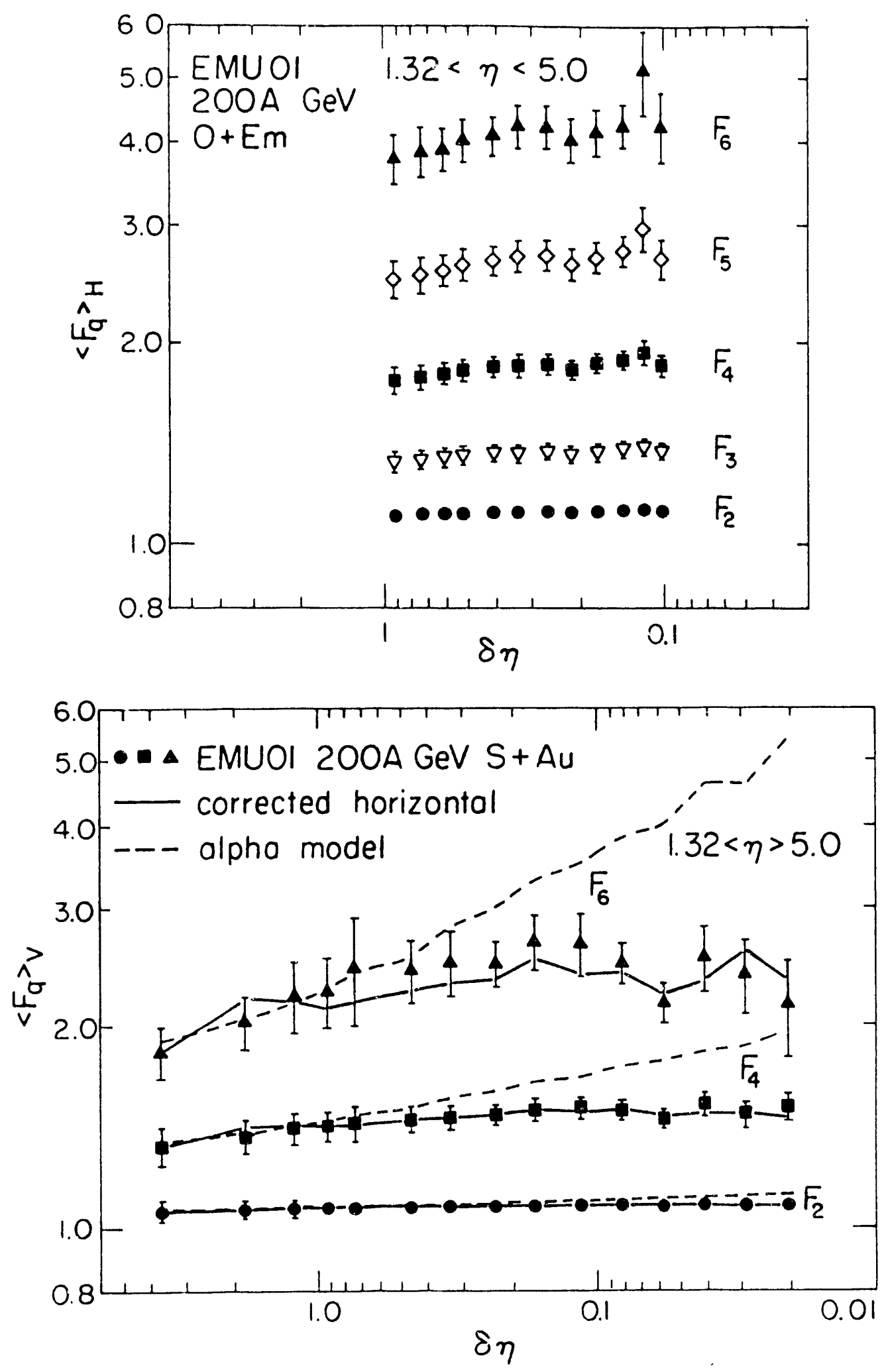

III-2. Intermittency analysis of central $\mathrm{S}+\mathrm{Au}$ collisions at $200 \mathrm{~A} \mathrm{GeV}$. Scaled factorial moments of order 2-6 are shown. Note that successive points are correlated, since each point represents a rebinning of the data from the point on its left. a) "Iforizontal" averaging, with $\delta \eta$ range cut off at 0.1 , as with most other experiments. b) EMU01 chamber data. with $\delta \eta$ range extended down to 0.01 due to higher resolution. For comparison, we show the "Alpha Model" with intermittency by definition, and corrected horizontal moments: see our paper (ref. 12) for details. 


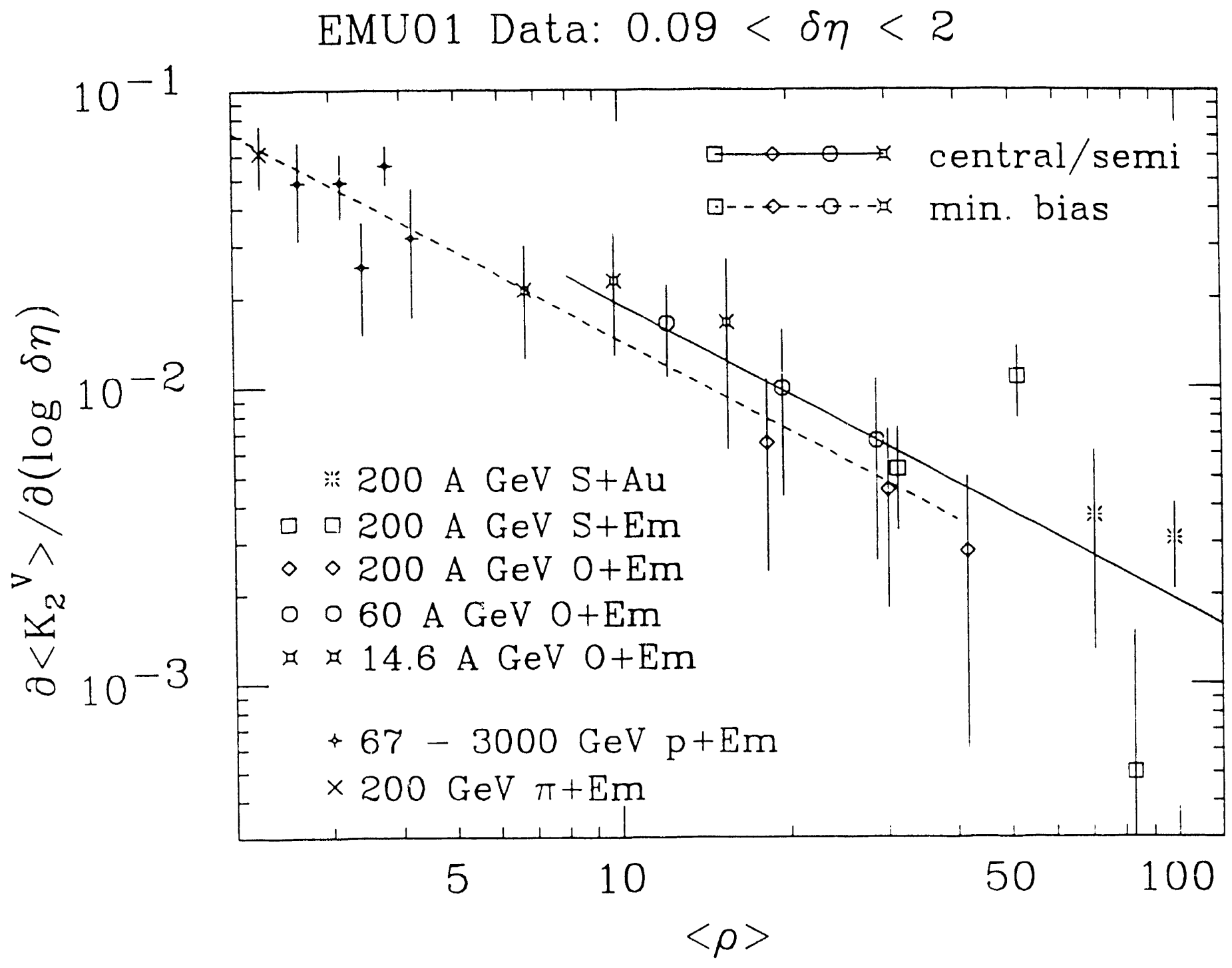

1II-3. Slopes of the factorial cumulant moments for the various data samples as a function of pseudorapidity density within a window 2 units wide centered upon the nucleon-nucleon center of mass rapidity. Note that here we plot the slope of $K_{q}$, not $\log K_{q}$, versus $\log \delta \eta$. The slopes are taken over the range $0.09<\delta \eta<2.0$. 
EMU01: 2-particle correlations

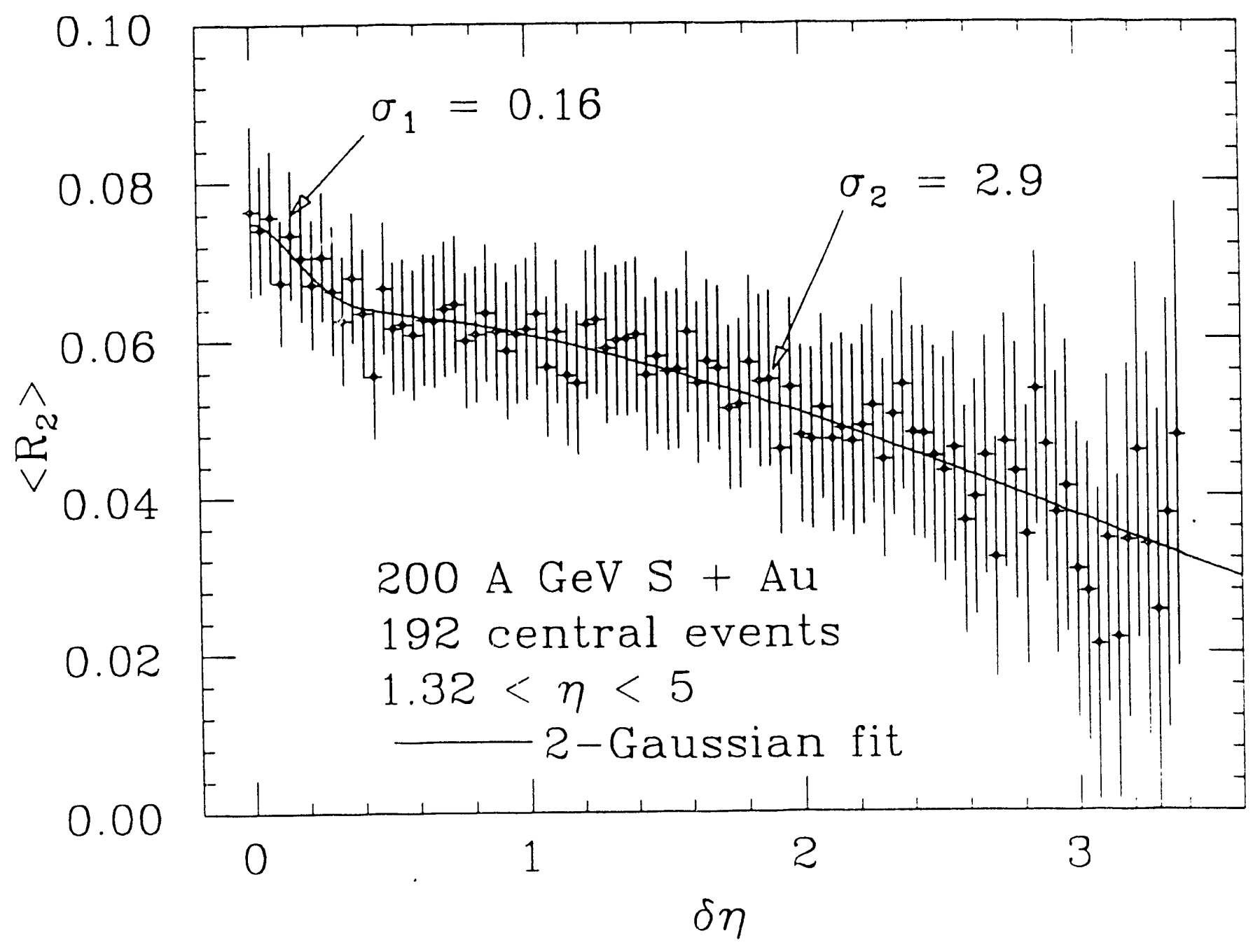

III-4. Two particle correlation function for the $S+A$ central data for the window $1.32<\eta<5.0$. The 2-gaussian fit is also shown. 


\section{EMU01 EMULSION CHAMBERS}

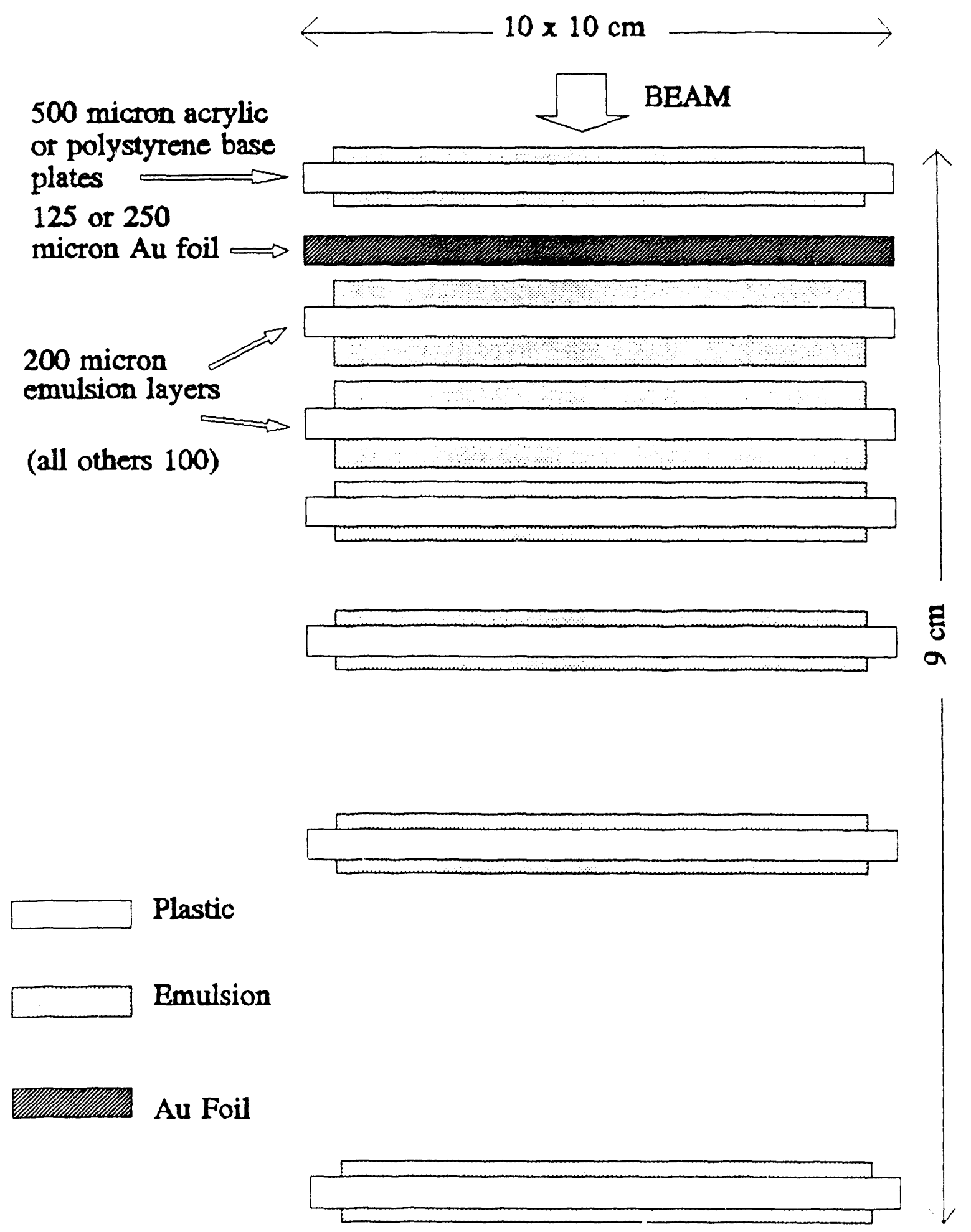

III-5. Typical emulsion chamber design used in EMU01/E815. 


\section{CAT/CAMS: SYSTEM HARDWARE}

Heidenhain digitizers

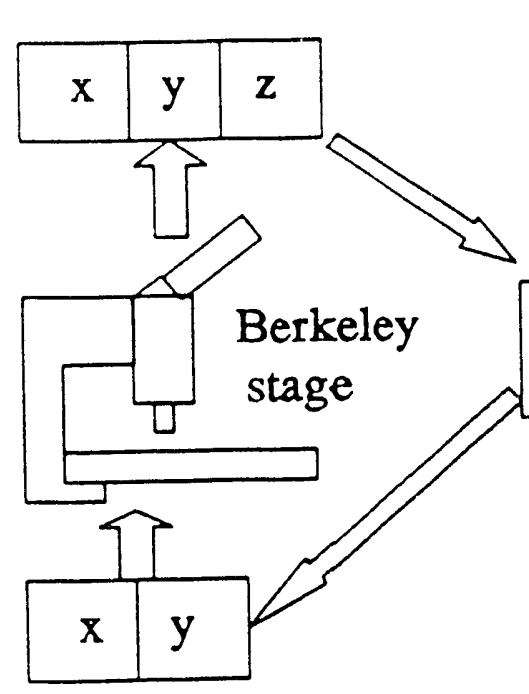

Galil servomotors

Digitizer precision: 0.5 microns

Positioning reproducibility: $\sim 5$ microns

\section{CAT/CAMS: SOFTWARE}

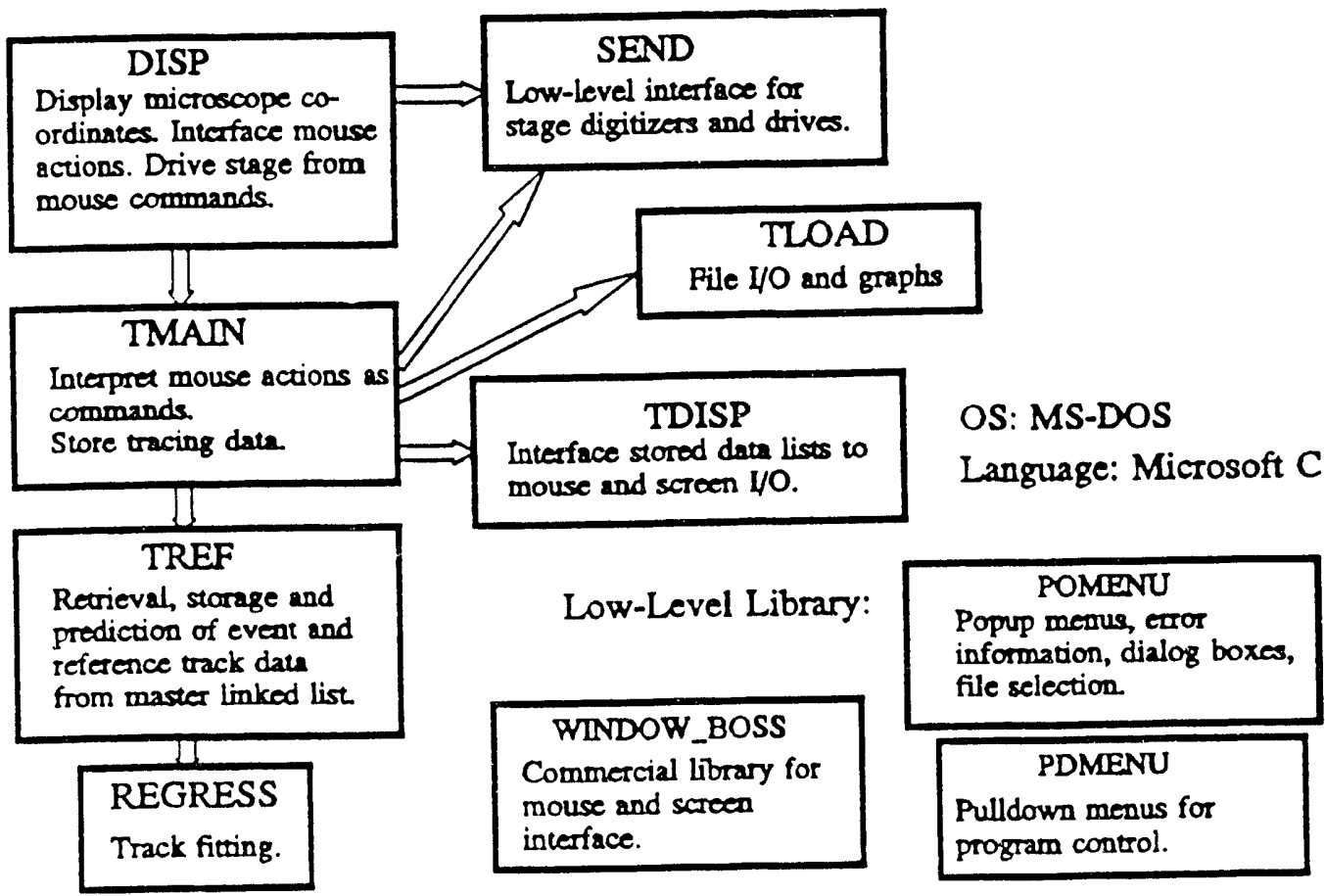

III-6. CAT/CAMS measuring system, implemented at UW. 

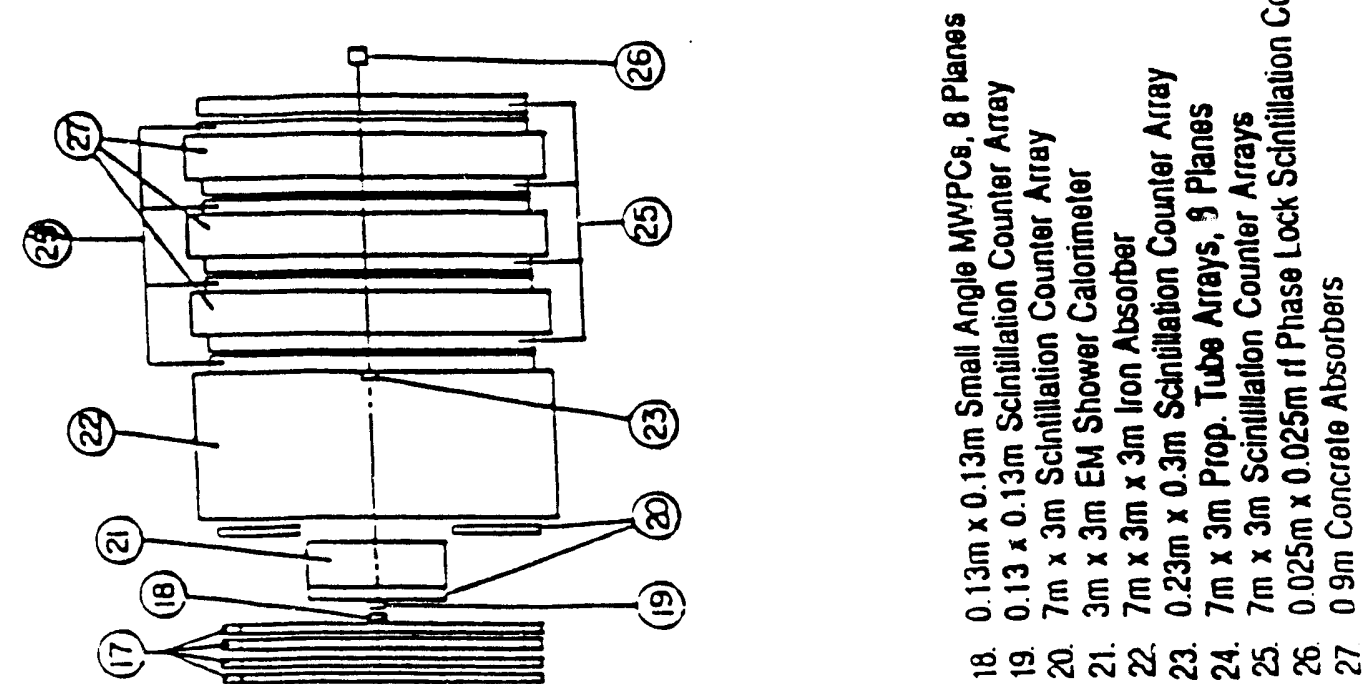

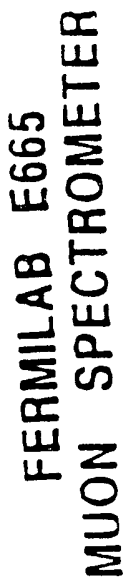
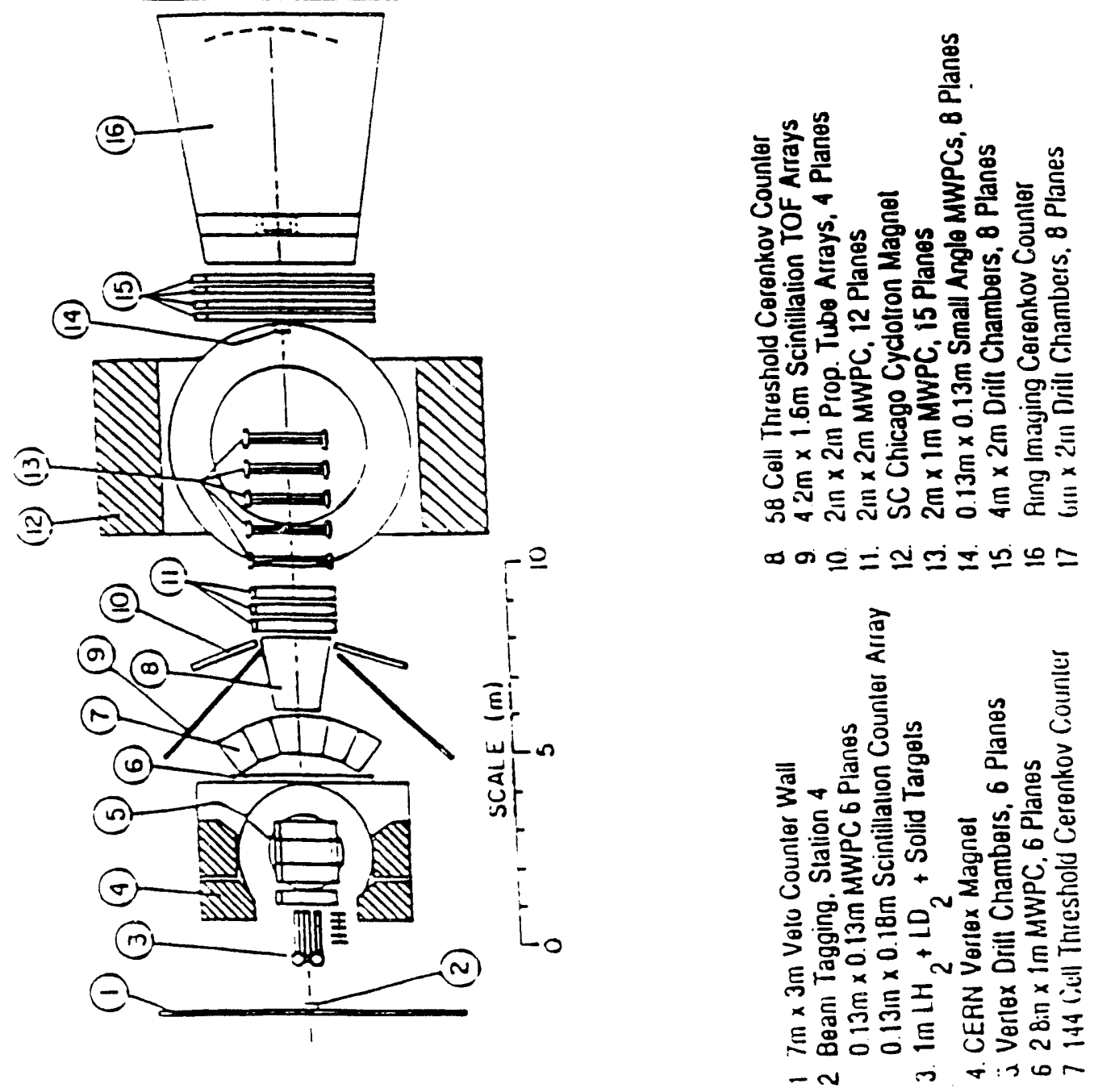

111-7. E665 deep inelastic muon scattering detector system. 
APPENDIX I: Publications During the Past Year

\section{Published Papers}

1. "DUMAND II Status Report," R.J. Wilkes, in High Energy Gamma Ray Astronomy, J. Matthews, ed., p. 285, AIP, New York, 1991.

2. "Evaluation of ACT Delay Lines for SSC/LHC Applications," R.J. Davisson, W.M. Dougherty, H.J. Lubatti, R.J. Wilkes, D. Fleisch, R. Kansy, G. Pieters, Nucl. Inst. and Meth. A295, 344 (1990).

3. "On the Multiplicity Fluctuations in Relativistic Heavy Ion Collisions," M.I. Adamovich, et al (EMU-01 Collaboration), Phys. Lett. B242(3,4), 512-516 (1990).

4. "Limiting Fragmentation, Scaling and Substructural Dependence of iviltiparticle Production in High Energy Ion Reactions," I. Otterlund, et al (EMU-01 Collaboration), Physica Scripta T\$2, 168 (1990).

5. "Proceedings of the DUMAND 1990 Trigger Workshop, July 12-13, 1990, Seattle, Washington," eds. K. Young and R. Wilkes.

6. "Scaled Factorial Moment Analysis of 200 A GeV Sulfur + Gold Interactions," R.J. Wilkes, et al (EMU01 Collaboration), in Proceedings of the Santa Fe Workshop, Intermittency in High Energy Collisions, Los Alamos National Laboratory, Los Alamos, USA, March 18-21, 1990, eds. F. Cooper, R.C. Hwa, I. Sarcevic, World Scientific, p. 145 (1991).

7. "Energy, Target and Multiplicity Dependence of Intermittency Behaviour in High-Energy $\mathrm{O}(\mathrm{Si}, \mathrm{S})$ Emulsion Interactions," M.I. Adamovich, et al (EMU-01 Collaboration), Z Phys C 49, 395 (1991).

8. "Stochastic Emission of Particles in Ultra- Relativistic Heavy-Ion Collisions," M.I. Adamovich, et al (EMU-01 Collaboration), Mod Phys Lett A 6, 469 (1991).

9. “On the Systematic Behaviour of the Intermittency-Indices in Nuclear Interactions, M.I. Adamovich, et al (EMU-01 Collaboration), LUIP 9011, to be published in Phys Lett B.

10. "Slow, Target Associated Particles Produced in Ultrarelativistic Heavy-Ion Interactions," M.I. Adamovich, et al (EMU-01 Collaboration), LUIP 9109, to be published in Phys Lett $B$.

11. “DUMAND-II Progress Report, " R.J. Wilkes, et al (DUMAND Collaboration), to be published in Proc. 4th Conference on Intersections between Particle and Nuclear Physics, Tucson, Arizona, May 1991.

12. "Fluctuaitons and Correlations in $200 A \mathrm{GeV} A-A$ Collisions," M.I. Adamovich, et al (EMU-01 Collaboration), whe published in Proc. Ith Conference on Intersections between Particle and Nuclear Physics, Tucson, Arizona, May 1991.

13. "Long-Duration Antarctic Balloon Flight Using Emulsion Chamber Cosmic Ray Detectors," R. J. Wilkes, J. Iwai, M. Wilber, J.H. Derrickson (for JACEE Collaboration) to be published in U.S. Antarctic Journal, 1991.

$$
\begin{array}{r}
\text { Publications that follow in this section have not bee } \\
\text { Appendix II begins on page } \\
\text { themoued and cyeled peparatily }
\end{array}
$$




\section{APPENDIX I: Collaboration Membership Lists}

A. List of Participants in DUMAND (Deep underwater muon and neutrino detection)

P. Bosetti, C. Ley, D. Samm, C. Wiebusch

Technische Hochschule Aachen, West Germany

P.K.F. Grieder, P. Minkowski

University of Bern, Switserland

S.T. Dye, E. Hazen

Boston University, USA

R. Becker-Szendy, J. Bolesta, J.G. Learned, S. Matsuno,

D. O'Connor, V.Z. Peterson, A. Roberts, V.J. Stenger

University of Hawaii, USA

P. Koske

University of Kiel, West Germany

T. Kitamura

Kinki University, Japan

K. Kobayakawa

Kobe University, Japan

H. Bradner

Scripps Institute of Oceanography, USA

T. Hayashino, M. Ito, H. Kawamoto, H. Susuki, S. Tanaka, A. Yamaguchi

Tohoku University, Japan

K. Mitsui, Y. Ohashi, A. Okada

Institute of Cosmic Ray Research, University of Tokyo, Japan

M. Sakuda, S. Uehara

KEK, Tsukuba, Japan

K. Miller, C.E. Roos, M. Webster

Vanderbilt University, USA

P.E. Boynton, J.J. Lord, R.J. Wilkes, K.K. Young

University of Washington, USA

U. Camerini, R. March

University of Wisconsin, USA 
B. List of Participants in Project JACEe (Balloon light experiment)

M.L. Cherry, W.V. Jones, Y. Tominaga, J.P. Wefel

Louisiana State Iniversity

J.H. Derrickson, W.F. Fountain, T.A. Parnell, E. Roberts, T. Tabuki, J. Watts NASA/MSFC

J.C. Gregory, T. Hayashi, Y. Takahashi

University of Alabama/Huntsville

T.H. Burnett, J. Iwai, J.J. Lord, S.C. Strausz, R.J.Wilkes

University of Washington

R. Holynski, A. Jurak, B. Wilczynska, H. Wilczynski, W. Wolter, B. Wosiek Institute of Nuclear Physics, Krakow

T. Shibata

Aoyama Gakuin University

S. Dake, H. Oda

Kobe University

M. Fuki

Matsusho Gakuen College

O. Miyamura

Osaka University

T. Ogata

University of Tokyo (Institute for Cosmic Ray Research) 
C. List of Participants in Experiments EMU-01/E-815 (Accelerator heavy ion interactions)

G.F. Xu, P.Y. Zheng

Institute of High Energy Physics, Beijing

K. Bhalla, A. Gill, G.L. Kaul, V. Kumar, P. Lal, S. Lokanathan, S. Mookerjee, R. Raniwala,

S. Raniwala, V.S. Shukla

University of Jaipur

A. Bhasin, S. Kachroo, L.K. Mangotra, N.K. Rao

University of Jammu

E.M. Friedländer, H.H. Heckman, P.J. Lindstrom

Lawrence Berkeley Laboratory

S. Garpman, B. Jakobsson, L. Karlsson, I. Otterlund, S. Persson, K. Söderström, E. Stenlund University of Lund

B. Dressel, E.R. Ganssauge, S. Hackel, H. Kallies, C. Mueller, J.T. Rhee, W. Schultz University of Marburg

B. Judek, R.S. Storey

National Research Council (Canada)

J. F. Sun, Z.Q. Weng, D.H. Zhang

Shanxi Normal University

S.A. Asimov, L.P. Chernova, S.I. Gadzhieva, K.G. Gulamov, F.G. Kadyrov, N.S. Lukicheva, V.S. Navotny, L.N. Svechnikova

Physical-Technical Institute of Tashkent

T.H. Burnett, J. Grote, T. Koss, J.J. Lord, D. Skelding, S. Strauss, R.J. Wilkes

University of Washington

X. Cai, L. S. Liu, W.Y. Qian, H.Q. Wang, D.C. Zhou, J.C. Zhou

Hua-Zhong Normal University, Wuhan

M.I. Adamovich, Y.A. Alexandrov, M.M. Chernyavsky, S.G. Gerassimov, S.P. Kharlamov, V.G. Larionova, N.V. Maslennikova, G.I. Orlova, N.G. Peresadko, N.A. Salmanova, M.I. Tretyakova Lebedev Institute of Physics, Mascow

M.M. Aggarwal, R. Arora, V.S. Bhatia, M. Kaur, I.S. Mittra

Panjab University

Z.V. Ameeva, N.P. Andreeva, Z.V. Anson, V.I. Bubnov, I.Y. Chasnicov, G.Z. Eligbaeva,

L.E. Eremenko, A.S. Gaitinov, G.S. Kalyachkina, E.K. Kanygina, T.I. Shakhova

Institute of High Energy Physics, Alma-Ata

E. Basova, R.A. Bondarenko, U.G. Gulyamov, S.H. Nasyrov, N.V. Petrov, N.A. Saidkhanov, T.P. Trofimova

Institute of Nuclear Physics, Tashkent

V.G. Bogdanov, V.A. Plyuschev

Khlopin Radium Inst., Leningrad 
D. List of Participants in Fermilab Experiment E665

(Deep inelastic muon interactions)

D. Geesaman, R. Gilman, M. Green, H. Jackson, S. Kaufman, S. Tentindo-Repond

Argonne National Laboratory

R. Kennedy, H. Kobrak, A. Salvarani, R. Swanson

University of California at San Diego

A. Eskreys, K. Eskreys, J. Figiel, D. Kisielewska, P. M 'scki, B. Pawlik

Institute of Nuclear Physics, and Institute of Nuclea hysics \& Technology

of the Academy of Mining and Engineering, Cracow, Poland

F. Bartlett, G. Coutrakon, J. Hanlon, T. Kirk, S. Krzywdzinski, H. Melanson, H. Montgomery,

J. Morfin, A. Osbourne, S. Wolbers

Fermi National Accelerator Laboratory

T. Dreyer, M. Erdmann, A. Haas, W. Mohr, H. Stier

University of Freiburg

J. Conrad, D. Michael, R. Nickerson, F. Pipkii, M. Schmitt, R. Wilson

Harvard University

M. Adams, C. Halliwell, S. Magill, D. McLeod, L. Sexton

University of nlinois at Chicago

S. Aid, S. Kunori, S. O'Day, E. Ramberg, A. Skuja, G. Snow, R. Talaga

University of Maryland

P. Anthony, M. Baker, W. Busza, T. Lyons, L. Osborne, J. Ryan

Massachuset ts Institute of Technology

I. Derado, V. Eckardt, H.J. Gebauer, G. Jansco, A. Manz, N. Schmitz, S. Soldner-Rembold, H.J. Seyerlein, P. Stopa, P. Strube, H.J. Trost, M. Vidal, W. Wittek, G. Wolf

Max Planck Institute for Physics \& Astrophysics, Munich

A. Bhatti, R. Davisson, W. Dougherty, D. Jansen, J. Lord, H. Lubatti, J. Wilkes, T. Zhao University of Washington

H. Braun, U. Ecker, A. Roeser

University of Wuppertal

S. Dhawan, V. Hughes, P. Schuier, H. Venkataramania

Yale University 

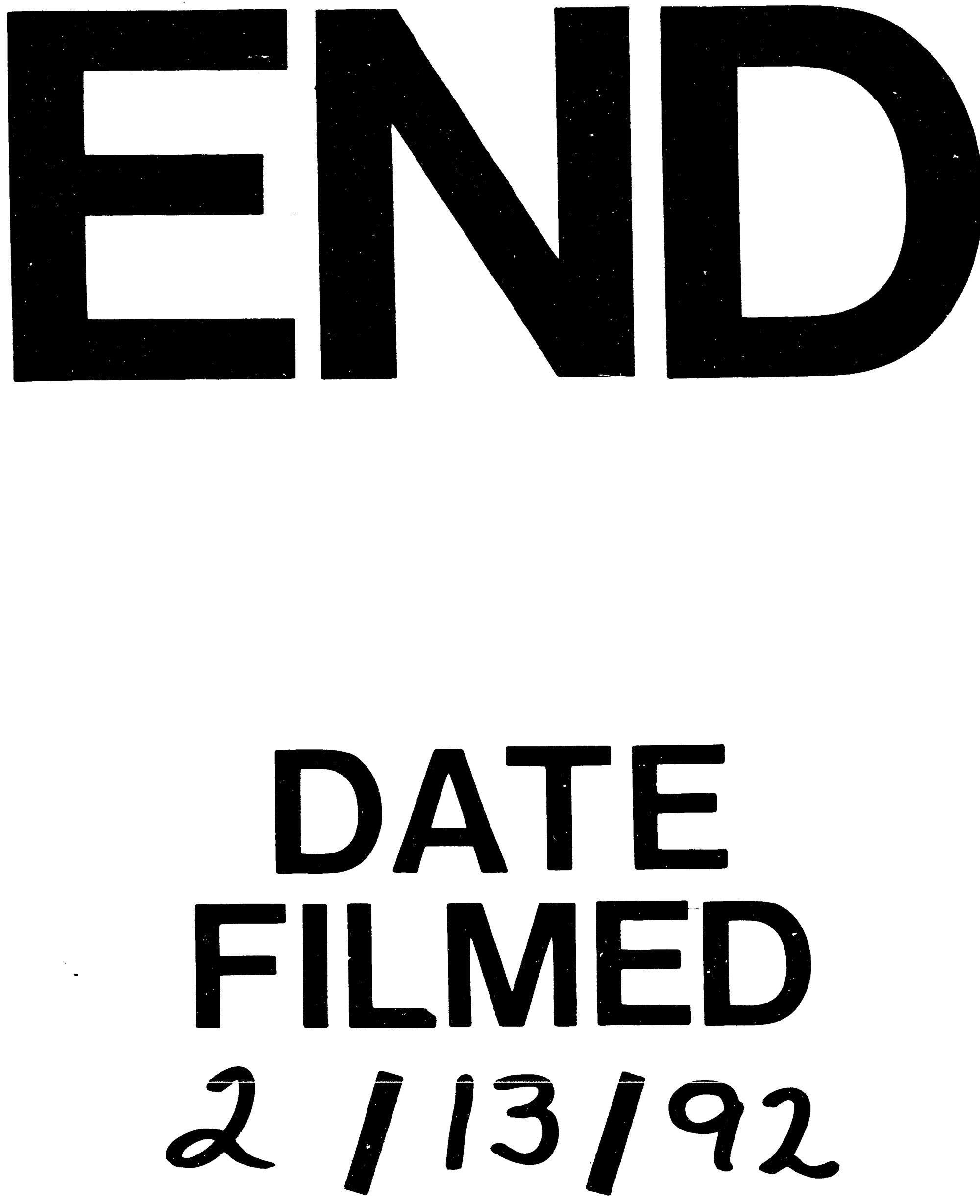
